\title{
Convergence and stability of a micro-macro acceleration method: linear slow-fast stochastic differential equations with additive noise
}

\author{
Przemysław Zieliński ${ }^{a}$, Hannes Vandecasteele ${ }^{\mathrm{a}}$, Giovanni Samaey ${ }^{\mathrm{a}}$ \\ ${ }^{a}$ KU Leuven, Department of Computer Science, NUMA Section, Celestijnenlaan 200A box 2402, 3001 Leuven, \\ Belgium
}

\begin{abstract}
We analyse the convergence and stability of a micro-macro acceleration algorithm for Monte Carlo simulations of linear stiff stochastic differential equations with a time-scale separation between the fast evolution of the individual stochastic realizations and some slow macroscopic state variables of the process. The micro-macro acceleration method performs a short simulation of a large ensemble of individual fast paths, before extrapolating the macroscopic state variables of interest over a larger time step. After extrapolation, the method constructs a new probability distribution that is consistent with the extrapolated macroscopic state variables, while minimizing Kullback-Leibler divergence with respect to the distribution available at the end of the Monte Carlo simulation. In the current work, we study the convergence and stability of this method on linear stochastic differential equations with additive noise, when only extrapolating the mean of the slow component. For this case, we prove convergence to the microscopic dynamics when the initial distribution is Gaussian and present a stability result for non-Gaussian initial laws.
\end{abstract}

Keywords: micro-macro acceleration methods, stiff stochastic differential equations, entropy minimization, Kullback-Leibler divergence, convergence \& stability

\section{Introduction}

Applications with multiple time scales arise in many domains, such as nanoscience [1], fluid dynamics [2], material science [3] and life sciences [4]. Still, the design and analysis of efficient numerical schemes for stiff stochastic differential equations (SDEs) remains challenging. On the one hand, due to a small stability domain, explicit schemes require too many time steps to reach the end of the simulation. On the other hand, while implicit methods are successful for ordinary differential equations, they fail to compute the correct invariant distribution for SDEs [5. Therefore, stiff SDEs require new dedicated numerical multiscale methods. A lot of work has already been done over the years, and we refer to the heterogeneous multiscale method [6, 7], equation-free techniques [8, 9], a homogenization method that constructs an approximate model for the slow components [10] and S-ROCK [11, 12] in particular, as starting points in the literature. In this work, we assume that the distinction between the slow and fast variables is readily given by the physical model. For the linear SDEs with additive noise under study, one could in principle perform an eigenvalue analysis on the drift matrix, and if there is a spectral gap, then there is a distinction between some slow and fast modes.

Recently, a micro-macro acceleration algorithm was introduced to accelerate the Monte Carlo simulation of SDEs with a time-scale separation between the fast individual stochastic paths and some slow macroscopic state variables of interest, which we define as expectations of some quantities

Email addresses: przemyslaw.zielinksi@cs.kuleuven.be (Przemysław Zieliński), hannes.vandecasteele@cs.kuleuven.be (Hannes Vandecasteele), giovanni.samaey@cs.kuleuven.be (Giovanni Samaey) 
of interest over the microscopic distributions [13]. Micro-macro acceleration connects the two levels of description of the process: individual paths of the SDE model on the microscopic level and the macroscopic state variables at the macroscopic level. The method alleviates the computational cost of a direct Monte Carlo simulation by interleaving a short bursts of Monte Carlo simulation with extrapolation of the macroscopic state variables over a larger time step. After extrapolation, the method constructs a new probability distribution by matching the last available distribution after the microscopic simulation with the extrapolated macroscopic state variables. Matching minimally perturbs the last available distribution after the microscopic simulation to make it consistent with the extrapolated state variables. Thus, in this approach, matching is inherently an optimization problem. There are many ways of measuring the difference between probability distributions. Following [14], we choose relative entropy or Kullback-Leibler divergence in the present paper, based on information theoretic considerations; for some other strategies see [13].

In this manuscript, we use the Euler-Maruyama method for the microscopic time integration. Although this time stepping method does not reproduce the exact time-invariant distribution of the linear SDEs with additive noise under study, the error of the microscopic time integrator is not dominant against the numerical error induced by extrapolation. Second, it has been shown in [5] that the implicit methods for SDEs can yield inaccurate results with larger time steps. Therefore, the aim of micro-macro acceleration is to obtain an accurate, fully explicit time integration method that can go beyond the stability domain of the Euler-Maruyama method.

A few fundamental properties of micro-macro acceleration have already been investigated. First, in [14, convergence was studied for general SDEs and for any time-scale separation. The analysis requires fixing an infinite hierarchy of macroscopic state variables that are used for extrapolation. It is then shown that convergence not only depends on taking the micro and extrapolation time steps to zero, but also on extrapolating an increasing number of macroscopic state variables as the extrapolation time step decreases. Second, in [15, the asymptotic numerical stability of the micromacro acceleration method was studied on a linear system of SDEs with Gaussian initial conditions. The stability criterion that was employed checks if the distributions obtained from the micro-macro acceleration method reach the equilibrium Gaussian distribution of the underlying microscopic integrator as the number of micro-macro time-steps tends to infinity. The analysis reveals that, when the slow and fast components of the system are decoupled, the maximal extrapolation time step is independent of the time-scale separation.

Convergence and stability are concerned with two limiting situations: convergence studies the method's behaviour at a fixed moment in time as the extrapolation time step tends to zero, whereas stability studies the method's behaviour for large time steps and long time horizons. Once both convergence and stability have been established, one can look at the appropriate selection of the extrapolation time step and the number of macroscopic state variables for accuracy and efficiency. In [16], we recently investigated the accuracy and efficiency of the micro-macro acceleration on slowfast systems, showing numerically that micro-macro acceleration can simultaneously take larger time steps than the microscopic time integrator, while obtaining a smaller error than approximate macroscopic models for the slow component of the system.

In this work, we expand the convergence and stability study of the micro-macro acceleration method on linear slow-fast SDEs with additive noise. For these equations, we prove convergence to the microscopic dynamics for Gaussian initial conditions when only extrapolating the mean of the slow component. To this end, we look at the propagation of the mean and variance of the full microscopic state throughout the micro-macro acceleration scheme, only extrapolating the mean of the slow component of the SDE. We prove that, when the extrapolation time step goes to zero, these two first moments converge to the corresponding ones produced by the underlying EulerMaruyama scheme. Dragging the microscopic time step to zero, we further obtain convergence to the exact dynamics of the slow-fast SDE. In contrast to the convergence result from [14], this 
analysis does not require any hierarchy of macroscopic state variables; both the mean of the fast component and the variance of the SDE are never extrapolated.

We also present a stability result for non-Gaussian initial laws that complements the previous findings in the Gaussian framework [15. For this part, we consider a class of (non-Gaussian) initial conditions having Gaussian tails. Inspired by the case of Gaussian initial conditions analyzed in [15], where the explicit formulas are available, we prove that the stability of the micro-macro acceleration method hinges on the stability of the mean obtained from the matching procedure. In this case, however, due to the non-Gaussianity of distributions, we have to look at the propagation of all higher moments throughout the method.

These findings illustrate a certain robustness of the matching procedure: solely using the first moment, matching reconstructs the remaining higher moments, so that we converge to the microscopic scheme as extrapolation vanishes, and recover its invariant distribution as the number of fixed extrapolations grows to infinity.

Slow-fast linear SDEs with additive noise. The objects of interest in this manuscript are linear SDEs with additive noise, or Ornstein-Uhlenbeck processes, of the form

$$
d X_{t}=A X_{t} d t+B d W_{t}
$$

with a square drift matrix $A \in \mathbb{R}^{d \times d}$, a diffusion matrix $B \in \mathbb{R}^{d \times d}$ and the Wiener process $W_{t} \in \mathbb{R}^{d}$. There are a few reasons why linear SDEs with additive noise are useful to study. First, the dynamics of linear systems is well understood, and we can derive stronger convergence results of micro-macro acceleration on such systems. Second, Ornstein-Uhlenbeck processes have an invariant distribution when all eigenvalues of $A$ have negative real part [17, p. 357]. We can then investigate whether micro-macro acceleration converges in distribution to the correct equilibrium distribution, as was done in [15] as a function of the time-scale separation and the extrapolation step size. Third, linear systems are popular in the context of ordinary differential equations to determine the stability of deterministic methods by an eigenvalue analysis of the drift matrix $A$. Although the concept of linearization is ambiguously defined in the stochastic case, linear SDEs with additive noise are useful to study in their own right.

In this manuscript, we are concerned with linear SDEs with a time-scale separation between some slow and some fast variables. A spectral gap in the drift matrix $A$ is a good indication of a time-scale separation present in the linear system. The larger the gap, the more stiff the linear system (1) becomes. In the context of micro-macro acceleration, we are mainly interested in the evolution of some moments of the slow components of (1). Using the spectral decomposition theorem, we introduce the orthogonal projections $\Pi^{s}: \mathbb{R}^{d} \rightarrow \mathbb{R}^{d_{s}}$ and $\Pi^{f}: \mathbb{R}^{d} \rightarrow \mathbb{R}^{d_{f}}$, with $d_{s}+d_{f}=$ $d$, that map the full state space onto the 'slow' $\mathbb{R}^{d_{s}}$ and 'fast' $\mathbb{R}^{d_{f}}$ state spaces, respectively, which correspond to the gap in the spectrum of $A$. This projection operation is also called 'coarsegraining' [15]. The decomposition is such that we can express the full state space and the drift matrix as

$$
\mathbb{R}^{d}=\mathbb{R}^{d_{s}} \oplus \mathbb{R}^{d_{f}}, \quad A=A^{s} \Pi^{s} \oplus A^{f} \Pi^{f},
$$

where the matrices $A^{s} \in \mathbb{R}^{d_{s} \times d_{s}}$ and $A^{f} \in \mathbb{R}^{d_{f} \times d_{f}}$ are the slow and fast drift terms respectively. We aim at accurately computing the moments of the projected slow process $\Pi^{s} X_{t}$ to approximate the exact evolution of these moments with a reasonable accuracy. For the remainder of the manuscript, a superscript ' $s$ ' denotes the slow components and a superscript ' $f$ ' the fast.

Outline of the paper. The paper is organized as follows: in Section 2 , we introduce the micromacro acceleration algorithm, specifically in our context of linear SDEs with additive noise. Section 3 contains the proof of convergence of micro-macro acceleration to the complete microscopic 
dynamics with only slow mean extrapolation. Section 4 investigates the stability of the method when the initial condition has Gaussian tails, when only extrapolating slow mean. In Section 5 . we illustrate the theoretical results on convergence and stability with numerical examples. We consider a system of linear SDEs with additive noise, with an extra periodic force on the slow component. We also compare the efficiency of micro-macro acceleration to existing acceleration and homogenization methods in Section 5 on a non-linear bimodal example. Section 6 contains a concluding discussion.

\section{The micro-macro acceleration algorithm}

One cycle of the micro-macro acceleration consists of four parts: (i) a microscopic simulation of the (stiff) stochastic differential equation over a short time interval, discretized with small time steps $\delta t$; (ii) restriction or computing an estimate of the macroscopic state variables based on the microscopic ensembles from (i); (iii) extrapolation of the restricted macroscopic state variables over a larger time step $\Delta t \gg \delta t$; (iv) matching the extrapolated state variables onto a probability distribution that perturbs the final distribution from (i) minimally.

Matching is the hardest step of the micro-macro acceleration algorithm. During matching, we build a new probability distribution that is consistent with the extrapolated state variables. This problem is often ill-posed, since there can be many probability distributions consistent with a given set of macroscopic state variables. Therefore, it was proposed in [13] to find the distribution that minimizes the divergence with respect to a prior distribution $P$. Such a prior is naturally available as the final distribution from the simulation step of the micro-macro acceleration method. In this work, we use matching introduced in [13, 14] and based on minimizing the Kullback-Leibler divergence (also called relative entropy)

$$
\mathcal{D}(Q \| P)=\mathbb{E}_{Q}\left[\ln \left(\frac{\mathrm{d} Q}{\mathrm{~d} P}\right)\right],
$$

over all distributions $Q$ that are consistent with the extrapolated states.

In this section, we first derive some explicit formulas for this matching procedure for linear slow fast SDEs (Section 2.1), after which we present the complete micro-macro acceleration method in full mathematical detail (Section 2.2. .

\subsection{Matching with the slow mean}

In this paper, we are particularly interested in the matching procedure that reconstructs a full microscopic distribution based only on the slow mean. For more general matching operators, see $[13,14]$. In this case, denoting by $\bar{\mu}^{s}$ the mean of the slow component, the matching reads

$$
\mathcal{M}\left(\bar{\mu}^{s}, P\right)=\underset{Q \in \mathscr{P}}{\arg \min } \mathcal{D}(Q \| P), \text { s.t. } \mathbb{E}_{Q}\left[\Pi^{s}\right]=\bar{\mu}^{s} .
$$

The distribution $\bar{Q}$ solving (2) is always absolutely continuous with respect to the prior $P$ and its density has exponential shape given by

$$
\frac{\mathrm{d} \bar{Q}}{\mathrm{~d} P}(x)=\exp \left(\bar{\lambda}^{s} \cdot x-A\left(\bar{\lambda}^{s}, P\right)\right),
$$

where the normalization constant (log-partition function) is $A\left(\lambda^{s}, P\right)=\ln \mathbb{E}_{P}\left[\exp \left(\lambda^{s} \cdot \Pi^{s}\right)\right]$. The optimal Lagrange multipliers $\bar{\lambda}^{s} \in \mathbb{R}^{d_{s}}$ are unique and fulfill [14]

$$
\nabla_{\lambda_{s}} A\left(\bar{\lambda}^{s}, P\right)=\bar{\mu}_{s} .
$$

In particular, when the prior $P$ has density $\pi_{P}$ with respect to the Lebesgue measure on $\mathbb{R}^{d}$, so does $\bar{Q}$ and its density reads

$$
\pi_{Q}(x)=\exp \left(\bar{\lambda}^{s} \cdot x-A\left(\bar{\lambda}^{s}, P\right)\right) \pi_{P}(x) .
$$


Moreover, when the prior $P$ is Gaussian, there is a closed expression for $\bar{Q}$, as will become clear in Lemma 1 (Section 3.1). When $P$ is not Gaussian, we need to resort to numerical methods to solve (3) for the Lagrange multipliers, see, e.g., [13].

The following result connects matching of the full microscopic distribution $P$ with a given slow mean $\bar{\mu}^{s}$ to the corresponding matching procedure using the slow marginal $P^{s}$ of the microscopic distribution as prior.

Proposition 1. Let $\bar{Q}=\mathcal{M}\left(\bar{\mu}^{s}, P\right)$ be the solution to 21 and $\bar{Q}^{s}=\mathcal{M}\left(\bar{\mu}^{s}, P^{s}\right)$ be the solution to the matching of slow prior marginal $P^{s}$. Then, the matching densities satisfy

$$
\frac{\mathrm{d} \bar{Q}}{\mathrm{~d} P}(y, z)=\frac{\mathrm{d} \bar{Q}^{s}}{\mathrm{~d} P^{s}}(y), \quad y \in \mathbb{R}^{d_{s}}, z \in \mathbb{R}^{d_{f}},
$$

and, in particular, all slow observables of $\bar{Q}$ equal the corresponding observables of $\bar{Q}^{s}$. Moreover, for any function $f$ on $\mathbb{R}^{d_{f}}$, the fast observable of $\bar{Q}$ generated by $f$ is given as

$$
\mathbb{E}_{\bar{Q}}[f(Z)]=\int_{\mathbb{R}^{d_{s}}} \mathbb{E}_{P}[f(Z) \mid Y=y] \bar{Q}^{s}(\mathrm{~d} y) .
$$

Proof. To see the first identity, consider the log-partition function $A(\lambda, P)$. Employing the decomposition of $P$ into its marginal and conditional [18, Thm. 10.2.1], we compute

$$
\begin{aligned}
A(\lambda, P) & =\ln \int_{\mathbb{R}^{d}} \exp \left(\lambda \cdot \Pi^{s} x\right) P(\mathrm{~d} x) \\
& =\ln \left\{\int_{\mathbb{R}^{d_{s}}} \exp (\lambda \cdot y) \int_{\mathbb{R}^{d_{f}}} P^{f \mid s}(\mathrm{~d} z \mid y) P^{s}(\mathrm{~d} y)\right\} \\
& =\ln \mathbb{E}_{P^{s}}\left[\exp \left(\lambda \cdot \Pi^{s}\right)\right]=A\left(\lambda, P^{s}\right),
\end{aligned}
$$

where $A\left(\lambda, P^{s}\right)$ is the log-partition function of the marginal prior $P^{s}$. Since both log-partition functions agree, the vector of Lagrange multipliers $\bar{\lambda} \in \mathbb{R}^{d_{s}}$ of $\bar{Q}$ corresponds exactly to the one of $\bar{Q}^{s}$. Therefore, we can write $\mathrm{d} \bar{Q} / \mathrm{d} P(y, z)=\exp \left(\bar{\lambda} \cdot y-A\left(\bar{\lambda}, P^{s}\right)\right)$, which proves the first identity.

To unwrap the fast mean of $\bar{Q}$, let us first write

$$
\mathbb{E}_{\bar{Q}}[f(Z)]=\int_{\mathbb{R}^{d}} f(z) \bar{Q}(\mathrm{~d} y, \mathrm{~d} z)=\int_{\mathbb{R}^{d}} f(z) \frac{\mathrm{d} \bar{Q}}{\mathrm{~d} P}(y, z) P(\mathrm{~d} y, \mathrm{~d} z) .
$$

Using the identity between the densities and [18, Thm. 10.2.1] once more, we arrive at

$$
\begin{aligned}
\mathbb{E}_{\bar{Q}}[f(Z)] & =\int_{\mathbb{R}^{d_{s}}} \int_{\mathbb{R}^{d_{f}}} f(z) \frac{\mathrm{d} \bar{Q}^{s}}{\mathrm{~d} P^{s}}(y) P^{f \mid s}(\mathrm{~d} z \mid y) P^{s}(\mathrm{~d} y) \\
& =\int_{\mathbb{R}^{d_{s}}}\left[\int_{\mathbb{R}^{d_{f}}} f(z) P^{f \mid s}(\mathrm{~d} z \mid y)\right] \frac{\mathrm{d} \bar{Q}^{s}}{\mathrm{~d} P^{s}}(y) P^{s}(\mathrm{~d} y),
\end{aligned}
$$

which concludes the proof of 4 .

\subsection{The complete micro-macro acceleration method}

In this section, we describe the four steps of the micro-macro acceleration algorithm in detail. We first present the time discretization of the linear SDE (1). We further introduce the restriction operator together with linear extrapolation of the macroscopic state variables. Finally, we use the matching operators as discussed in the previous section.

Let $P_{n}$ be the probability distribution at time $t_{n}=n \Delta t$. The micro-macro acceleration algorithm advances the distribution $P_{n}$ to a distribution $P_{n+1}$ at time $t_{n+1}=t_{n}+\Delta t$ in four stages:

Step 1: Microscopic time integration. In the first step, we perform a simulation of (1) over a time window of size $\delta \tau$. In practice, we usually take $K$ time steps of size $\delta t$, such that $\delta \tau=K \delta t$. In this text, we use the Euler-Maruyama scheme to discretize (1), reading

$$
X_{n, k+1}=(I+A \delta t) X_{n, k}+\sqrt{\delta t} B \delta W_{n, k},
$$

for $k=0, \ldots, K-1$, and where $\delta W_{n, k}$ are Brownian increments. The random variables $X_{n, k}, k=$ $0, \ldots, K$ have probability distributions $P_{n, k}$ and we denote the initial distribution as $P_{n, 0}=P_{n}$. 
Step 2: Restriction. Second, to transition from the full microscopic description to the macroscopic state variables, we compute the mean of the slow component of the process $(1)$, reading $\mathbb{E}_{P}\left[\Pi^{s}\right]$. We restrict the slow mean at every microscopic time step, generating a sequence of values of the slow mean:

$$
\mu_{n, k}^{s}=\mathbb{E}\left[\Pi^{s} P_{n, k}\right], \quad k=0, \ldots, K .
$$

Step 3: Extrapolation. In the third step, we perform time integration on the macroscopic level over a time interval of size $\Delta t$. Given the slow means $\mu_{n, k}^{s}, k=0, \ldots, K$ at times $t_{n}+k \delta t$ from the previous step, we compute the slow mean $\mu_{n+1}^{s}$ at time $t_{n+1}=t_{n}+\Delta t$ by linear extrapolation

$$
\mu_{n+1}^{s}=\mu_{n}^{s}+\frac{\Delta t}{K \delta t}\left(\mu_{n, K}^{s}-\mu_{n}^{s}\right)
$$

Note that we only use the state variables at time $t_{n}$ and $t_{n}+K \delta t$.

Step 4: Matching. Finally, we construct a new probability distribution that is consistent with $\mu_{n+1}^{s}$. To this end, we employ the matching operator (2) and define

$$
P_{n+1}=\mathcal{M}\left(\mu_{n+1}^{s}, P_{n, K}\right)
$$

to obtain a new microscopic distribution $P_{n+1}$ at time $t_{n+1}=t_{n}+\Delta t$. The prior distribution $P_{n, K}$ is the final distribution computed during Step 1.

Remark 1 (On choosing the time-stepping parameters). The first criterion for the choice of $\delta t$ and $\Delta t$ must be based on stability considerations. To ensure stability of the Euler-Maruyama scheme, we need $\delta t$ such that, when $A$ has all eigenvalues negative, the spectral radius $\rho(I+\delta t A)$ is smaller than 1 . To guarantee this, it suffices to choose $\delta t$ such that $\delta t<2 / \rho(A)=2 / \rho\left(A^{f}\right)$, due to the spectral gap of $A$. Similarly, because the linear extrapolation concerns only the slow mean, we can expect that it is enough to have $\Delta t \lesssim 1 / \rho\left(A^{s}\right)$. We give more substance to this intuition in Section 4 .

Having ensured stability, the crucial aspect in selecting particular values of microscopic time window $\delta \tau=K \delta t$ and the macroscopic step $\Delta t$ is that of accuracy we want to obtain. To keep things simple, we illustrate their effect on the local error in the extrapolation of the slow mean and refer to Section 3 for the analysis of matching influence. If we denote by $\mu_{t}^{s, e}$ the exact slow mean of equation (1), by employing Euler-Maruyama scheme we make first order error in approximating $\mu_{t}^{s, e}$ on the microscopic time window, i.e.

$$
\left\|\mu_{t_{n}+\delta \tau}^{s, e}-\mu_{n, K}^{s}\right\| \leq C(\delta \tau) \delta t
$$

where $C(\delta \tau)$ is a positive constant that depends on the coefficients of (1). Therefore, comparing the Taylor expansion of $\mu_{t_{n+1}}^{s, e}$ around $t=t_{n}$ to the extrapolated slow mean (6) we get

$$
\left\|\mu_{t_{n+1}}^{s, e}-\mu_{n+1}^{s}\right\| \leq \Delta t[C(\delta \tau) / K+\mathcal{O}(\delta t)]+\mathcal{O}\left((\Delta t)^{2}\right)
$$

Thus, we can see that the error vanishes linearly with macroscopic step $\Delta t$ and the constant depends only on the parameters of microscopic time integration.

Let us also mention that the number of microscopic time steps $K$ is closely related to the order of extrapolation procedure we want to employ in Step 3. For the linear extrapolation (6), it suffices to take $K=1$, and this is what we do in the rest of the manuscript. However, having $K>1$ allows to replace (6) with other procedures that use intermediate values of the slow mean $\mu_{n, 1}^{s}, \ldots, \mu_{n, K-1}^{s}$ directly in the computation of $\mu_{n+1}^{s}$, for example polynomial extrapolation [19]. Such higher order extrapolation could result in having $(\Delta t)^{K}$ instead of $\Delta t$ in the estimate above. 


\section{Convergence of the micro-macro acceleration method with slow mean extrapolation}

In this section, we prove that the micro-macro acceleration method of Section 2 converges to the exact dynamics of the linear SDE (1), when only extrapolating the mean of the slow process and when the initial condition is Gaussian (Theorem 1 in Section 3.2). Before proceeding to the proof, we need an intermediate result, that explicitly describes the evolution of the mean and variance of the full microscopic system under the micro-macro acceleration method. This intermediate result is the subject of Section 3.1. Theorem 1 differs from the main convergence result in [14], as the latter requires a hierarchy of macroscopic state variables to form a complete description of the density it represents. The slow mean by itself never forms such a complete description of the underlying density. An extension of Theorem 1 to non-linear SDEs or non-Gaussian initial conditions is highly non-trivial.

\subsection{An iterative formula for slow mean-only extrapolation}

The proof of the convergence result in Theorem 1 relies on an iterative formula that describes how the complete mean and variance propagate through one step of the micro-macro acceleration scheme. As the micro-macro method preserves the Gaussianity of the initial condition, which we show below, the knowledge of the mean and variance suffices to control the distribution throughout the whole simulation. The derivation here assumes only one Euler-Maruyama inner step of size $\delta t$ for simplicity, but can easily be extended to $K$ inner steps.

We start with a lemma, proven also in [15] but by different means, that gives the matched distribution when the prior is Gaussian and we only match with the slow mean.

Lemma 1. Suppose $P$ is the prior Gaussian distribution with vector mean $\mu$ and a positive-definite covariance matrix $\Sigma$,

$$
\mu=\left[\begin{array}{l}
\mu^{s} \\
\mu^{f}
\end{array}\right], \quad \Sigma=\left[\begin{array}{cc}
\Sigma^{s} & C \\
C^{T} & \Sigma^{f}
\end{array}\right] .
$$

The distribution $\bar{Q}=\mathcal{M}\left(\bar{\mu}^{s}, P\right)$, which solves (2), is also Gaussian with the same variance and mean $\bar{\mu}=\left[\bar{\mu}^{s}, \bar{\mu}^{f}\right]^{T}$ where $\bar{\mu}^{f}=\mu^{f}+C^{T}\left(\Sigma^{s}\right)^{-1}\left(\bar{\mu}^{s}-\mu^{s}\right)$.

Proof. Let $\bar{Q}^{s}=\mathcal{M}\left(\bar{\mu}^{s}, P^{s}\right)$, where $P^{s}$ is the slow marginal of $P$. Since $P^{s}=\mathcal{N}\left(\mu^{s}, \Sigma^{s}\right)$, by the Gaussianity of $P$, a standard result for Kullback-Leibler minimization states that $\bar{Q}^{s}$ is also Gaussian with the mean $\bar{\mu}^{s}$ and the same variance $\Sigma^{s}$. That $\bar{Q}$ is Gaussian and its slow variance equals $\Sigma^{s}$ follows directly from the expression connecting the Radon-Nikodym derivatives in Proposition 1

To compute the fast mean $\bar{\mu}^{f}$ and variance $\bar{\Sigma}^{f}$ of $\bar{Q}$, we use the second part of Proposition 1 . Focusing on $\bar{\mu}^{f}$ first, employing formula (4) with $f(z)=z$ and a well-known expression for the conditional mean for Gaussian distributions, we can write the matched fast mean as

$$
\bar{\mu}^{f}=\int_{\mathbb{R}^{d_{s}}}\left\{\mu^{f}+C^{T}\left(\Sigma^{s}\right)^{-1}\left(y-\mu^{s}\right)\right\} \bar{Q}^{s}(d y)=\mu^{f}+C^{T}\left(\Sigma^{s}\right)^{-1}\left(\bar{\mu}^{s}-\mu^{s}\right) .
$$

Similarly, we can express the fast matched variance by choosing $f(z)=\left(z-\bar{\mu}^{f}\right)\left(z-\bar{\mu}^{f}\right)^{T}$ in (4)

$$
\bar{\Sigma}^{f}=\int_{\mathbb{R}^{d_{s}}} \mathbb{E}_{P}\left[\left(Z-\bar{\mu}^{f}\right)\left(Z-\bar{\mu}^{f}\right)^{T} \mid Y=y\right] \bar{Q}^{s}(d y) .
$$

By adding and subtracting the fast conditional mean $\mu^{f \mid s}(y)=\mu^{f}+C^{T}\left(\Sigma^{s}\right)^{-1}\left(y-\mu^{s}\right)$ to each $Z-\bar{\mu}^{f}$, we get

$$
\bar{\Sigma}^{f}=\int_{\mathbb{R}^{d_{s}}}\left\{\mathbb{E}_{P}\left[\left(Z-\mu^{f \mid s}(y)\right)\left(Z-\mu^{f \mid s}(y)\right)^{T} \mid Y=y\right]+\left(\mu^{f \mid s}(y)-\bar{\mu}^{f}\right)\left(\mu^{f \mid s}(y)-\bar{\mu}^{f}\right)^{T}\right\} \bar{Q}^{s}(d y) .
$$


The first summand under the integral represents the fast conditional variance of $P$ and, since $P$ is Gaussian, it is independent of $y$ and equals $\Sigma^{f}-C^{T}\left(\Sigma^{s}\right)^{-1} C$. The second summand can be expanded using the expressions for $\mu^{f \mid s}(y)$ and $\bar{\mu}^{f}$. Writing it out, we obtain

$$
\begin{aligned}
\bar{\Sigma}^{f} & =\Sigma^{f}-C^{T}\left(\Sigma^{s}\right)^{-1} C+\int_{\mathbb{R}^{d_{s}}} C^{T}\left(\Sigma^{s}\right)^{-1}\left(y-\bar{\mu}^{s}\right)\left(y-\bar{\mu}^{s}\right)^{T}\left(\Sigma^{s}\right)^{-1} C \bar{Q}^{s}(d y) \\
& =\Sigma^{f}-C^{T}\left(\Sigma^{s}\right)^{-1} C+C^{T}\left(\Sigma^{s}\right)^{-1} \Sigma^{s}\left(\Sigma^{s}\right)^{-1} C=\Sigma^{f}
\end{aligned}
$$

where we used the fact that the slow variance of $\bar{Q}^{s}$ equals $\Sigma^{s}$.

With Lemma 1, we are armed to obtain a closed expression for the time-discrete evolution of the mean and variance of the slow-fast SDE (1), as generated by the micro-macro acceleration method of Section 2. When the initial condition is Gaussian with a positive definite covariance matrix, all intermediate distributions are Gaussian as well, a key property for the proof of Theorem 11. We can also relate the mean and covariance of the intermediate distributions to the mean and variance at the previous time instant. We summarize these results in Proposition 2 to make our assumptions clear.

Proposition 2. Suppose that the drift matrix $A$ is given in block form

$$
A=\left[\begin{array}{cc}
A^{s} & V \\
W & A^{f}
\end{array}\right],
$$

that the matrix $B$ is non-singular, and that the microscopic time step $\delta t$ is such that the matrix $A+\delta t I$ is also non-singular. If at time $t_{n}=n \Delta t$ the micro-macro distribution is Gaussian with mean

$$
\mu_{n}=\left[\begin{array}{l}
\mu_{n}^{s} \\
\mu_{n}^{f}
\end{array}\right],
$$

and with a positive-definite covariance matrix $\Sigma_{n}$, the probability distribution after one micromacro step, at time $t_{n+1}=t_{n}+\Delta t$, is also Gaussian with mean given by equation (10) and with covariance $\Sigma_{n, 1}$, given by one step of the Euler-Maruyama inner integrator.

Remark 2 (On the time step $\delta t$ ). The condition that $A+\delta t I$ is non-singular is equivalent to requiring that $-1 / \delta t$ is not an eigenvalue of $A$. A sufficient condition for the latter requirement to hold is when $1 / \delta t>\rho(A)=\|A\|$. This condition is only slightly stronger than the stability condition as stated in Remark 1. In practice, we would indeed need $1 / \delta t>\|A\|$ for accurate computational results. Hence, in our convergence study, when we let $\delta t$ decrease to $0, A+\delta t I$ is non-singular as long as $\delta t<\|A\|^{-1}$.

Proof. We can write each of the four steps of the algorithm in explicit form. First, we consider the microscopic simulation step. During one Euler-Maruyama step, a Monte Carlo particle $X_{n}$ is propagated as

$$
X_{n, 1}=(I+A \delta t) X_{n}+\sqrt{\delta t} B \xi_{n}, \quad \xi_{n} \sim \mathcal{N}^{d}(0,1),
$$

where $\mathcal{N}^{d}(0,1)$ denotes the $d$-dimensional standard normal distribution. The distribution of $X_{n, 1}$ is also Gaussian since $B$ is non-singular [20, Theorem 4.2]. Next, we perform the restriction step. By taking expectations, the mean $\mu_{n, 1}$ and the covariance $\Sigma_{n, 1}$ after the Euler-Maruyama step read

$$
\mu_{n, 1}=(I+A \delta t) \mu_{n}, \quad \Sigma_{n, 1}=(I+\delta t A) \Sigma_{n}(I+\delta t A)^{T}+\delta t B B^{T},
$$

after which we extrapolate the slow mean as

$$
\begin{aligned}
\mu_{n+1}^{s} & =\mu_{n}^{s}+\frac{\Delta t}{\delta t}\left(\mu_{n, 1}^{s}-\mu_{n}^{s}\right) \\
& =\mu_{n}^{s}+\frac{\Delta t}{\delta t}\left(\mu_{n}^{s}+\delta t A^{s} \mu_{n}^{s}+\delta t V \mu_{n}^{f}-\mu_{n}^{s}\right) \\
& =\left(I^{s}+\Delta t A^{s}\right) \mu_{n}^{s}+\Delta t V \mu_{n}^{f}
\end{aligned}
$$


Now that we have the extrapolated slow mean, we can use Lemma 1 to explicitly obtain the result of matching. Note that, since $I+\delta t A$ is invertible and $B B^{T}$ positive-definite, the covariance $\Sigma_{n, 1}$ obtained from $\Sigma_{n}$ via (7) remains positive-definite. As a consequence, the slow covariance matrix $\Sigma_{n, 1}^{s}$, as a principal sub-matrix of $\Sigma_{n, 1}$, is positive definite as well [21, Prop. 7.1.2]. According to Lemma 1, the matched distribution is also Gaussian when only extrapolating the slow mean. Furthermore, the covariance matrix is not affected by matching, i.e., $\Sigma_{n+1}=\Sigma_{n, 1}$, and the fast mean is given by

$$
\begin{aligned}
\mu_{n+1}^{f} & =\mu_{n, 1}^{f}+C_{n, 1}^{T}\left(\Sigma_{n, 1}^{s}\right)^{-1}\left(\mu_{n+1}^{s}-\mu_{n, 1}^{s}\right) \\
& =\delta t W \mu_{n}^{s}+\left(I^{f}+\delta t A^{f}\right) \mu_{n}^{f}+C_{n, 1}^{T}\left(\Sigma_{n, 1}^{s}\right)^{-1}\left((\Delta t-\delta t) A^{s} \mu_{n}^{s}+(\Delta t-\delta t) V \mu_{n}^{f}\right) \\
& =\left[\delta t W+(\Delta t-\delta t) C_{n, 1}^{T}\left(\Sigma_{n, 1}^{s}\right)^{-1} A^{s}\right] \mu_{n}^{s}+\left[I^{f}+\delta t A^{f}+(\Delta t-\delta t) C_{n, 1}^{T}\left(\Sigma_{n, 1}^{s}\right)^{-1} V\right] \mu_{n}^{f} .
\end{aligned}
$$

Bundling the propagation of the slow (8) and fast matched mean (9) in one vector $\mu_{n+1}$ gives

$$
\left[\begin{array}{c}
\mu_{n+1}^{s} \\
\mu_{n+1}^{f}
\end{array}\right]=\left[\begin{array}{cc}
I^{s}+\Delta t A^{s} & \Delta t V \\
\delta t W+(\Delta t-\delta t) C_{n, 1}^{T}\left(\Sigma_{n, 1}^{s}\right)^{-1} A^{s} & I^{f}+\delta t A^{f}+(\Delta t-\delta t) C_{n, 1}^{T}\left(\Sigma_{n, 1}^{s}\right)^{-1} V
\end{array}\right]\left[\begin{array}{c}
\mu_{n}^{s} \\
\mu_{n}^{f}
\end{array}\right] .
$$

To conclude, the time-discrete evolution of the mean of the slow-fast SDE (1), as generated by the micro-macro acceleration method is given by the time-dependent linear system (10), with initial condition equal to the mean of the initial distribution of (1).

\subsection{Convergence theorem}

All elements are now in place to prove convergence of the micro-macro acceleration method of Section 2 that only extrapolates the slow mean of the process. Our aim is to demonstrate that by appropriately reducing time steps the method, and the matching in particular, can reproduce the full microscopic laws with any level of accuracy and do not introduce modeling errors. To this end, we look at the time marginals of the laws generated by the method and prove that they converge to the corresponding laws of the exact solution to (1). We measure the quality of approximation with the Kullback-Leibler divergence (which in the Gaussian setting reduces to divergence between means and variances). The proof makes use of formula [10, and holds for general linear SDEs with additive noise.

Theorem 1. Given a linear additive noise SDE with a Gaussian initial distribution, consider the micro-macro acceleration algorithm with relative-entropy matching and slow-mean extrapolation. Also, fix an end time $T>0$. Denote by $P_{T}$ the exact distribution of the linear SDE at time $T$, and by $P_{n_{\Delta t}(T)}$ the distribution obtained using $n_{\Delta t}(T)$ steps of the micro-macro acceleration scheme of Section 2 with $K=1$, where $n_{\Delta t}(T)=\lfloor T / \Delta t\rfloor$. Then, under the assumptions of Proposition 2

$$
\lim _{\delta t \rightarrow 0} \lim _{\Delta t \rightarrow \delta t} \mathcal{D}\left(P_{n_{\Delta t}(T)} \| P_{T}\right)=0 .
$$

As a consequence, the distributions $P_{n_{\Delta t}(T)}$ obtained by micro-macro acceleration at time $T$ converge in total variation to the exact microscopic distribution $P_{T}$ in the same limits.

Proof. Since the initial condition is Gaussian, all intermediate distributions of the exact solution, the Euler-Maruyama method, and micro-macro acceleration are Gaussian too. By a standard expression for the Kullback-Leibler divergence between two Gaussian distributions [22, the divergence in 111 becomes

$\mathcal{D}\left(P_{n_{\Delta t}(T)} \| P_{T}\right)=\frac{1}{2}\left(\ln \frac{\left|\Sigma_{T}\right|}{\left|\Sigma_{n_{\Delta t}(T)}\right|}-d+\operatorname{Tr}\left(\Sigma_{T}^{-1} \Sigma_{n_{\Delta t}(T)}\right)+\left(\mu_{T}-\mu_{n_{\Delta t}(T)}\right)^{T} \Sigma_{T}^{-1}\left(\mu_{T}-\mu_{n_{\Delta t}(T)}\right)\right)$, 
where $\mu_{T}$ and $\Sigma_{T}$ are the mean and variance of $X_{T}$, and $\mu_{n_{\Delta t}(T)}$ and $\Sigma_{n_{\Delta t}(T)}$ are the mean and variance of $X_{n_{\Delta t}(T)}$.

First, we fix $\delta t \leq \Delta t$ and let $\Delta t$ decrease to $\delta t$. If we perform back-substitution in equation (10) to write the mean at time $T$ as a function of the initial mean vector, we obtain a product of $n_{\Delta t}(T)$ different matrices. The number of matrices increases to $n_{\delta t}(T)$ as $\Delta t$ decreases to $\delta t$, but there always remain a finite number of matrices because $\delta t>0$. The contribution of the largest off-diagonal term in (10) also reduces to zero and as a result, the mean vector $\mu_{n_{\Delta t}(T)}$ approaches the respective mean $\mu_{n_{\delta t}(T)}$ of the Euler-Maruyama scheme. We obtain

$$
\lim _{\Delta t \rightarrow \delta t}\left[\begin{array}{l}
\mu_{n(\Delta t)}^{s} \\
\mu_{n(\Delta t)}^{f}
\end{array}\right]=\left[\begin{array}{cc}
I^{s}+\delta t A^{s} & \delta t V \\
\delta t W & I^{f}+\delta t A^{f}
\end{array}\right]^{n(\delta t)}\left[\begin{array}{l}
\mu_{0}^{s} \\
\mu_{0}^{f}
\end{array}\right],
$$

where $\mu_{0}^{s}$ and $\mu_{0}^{f}$ are the slow and fast mean respectively of the initial condition. Similarly, since the variance stays constant during matching, $\Sigma_{n_{\Delta t}(T)}$ converges to $\Sigma_{n_{\delta t}(T)}$, the variance of the Euler-Maruyama scheme. Hence, the limit (11) reduces to

$$
\lim _{\delta t \rightarrow 0} \frac{1}{2}\left(\ln \frac{\left|\Sigma_{T}\right|}{\left|\Sigma_{n_{\delta t}(T)}\right|}-d+\operatorname{Tr}\left(\Sigma_{T}^{-1} \Sigma_{n_{\delta t}(T)}\right)+\left(\mu_{T}-\mu_{n_{\delta t}(T)}\right)^{T} \Sigma_{T}^{-1}\left(\mu_{T}-\mu_{n_{\delta t}(T)}\right)\right),
$$

which is the relative entropy between the Euler-Maruyama scheme and the exact solution at time $T$. This expression converges to zero because, as $\delta t$ decreases to zero, the mean and variance of the Euler-Maruyama method converge to their respective values of the exact solution at time $T$, by weak convergence [23, Thm. 14.1.5]. The conditions of the latter theorem are met since all moments of $P_{T}$ exist by Gaussianity of $P_{T}$ and the drift and diffusion terms of the linear additive noise SDE have sufficient regularity. Finally, by Pinsker's inequality, $P_{n_{\Delta t}(T)}$ converges to $P_{T}$ in total variation.

Theorem 1 might be surprising, since it does not require the number of macroscopic state variables to increase to infinity as the extrapolation time step $\Delta t$ decreases; using only the slow mean as a macroscopic state variable is sufficient. However, the result only holds for linear additive noise SDEs with Gaussian initial conditions, as the proof relies heavily on iteration 10 . At the moment, no proof exists on convergence for non-linear SDE with any (fixed) finite number of macroscopic state variables.

\section{Stability of micro-macro acceleration with initial condition with Gaussian tails}

In this section, we study the stability of micro-macro acceleration when applied to (1), i.e., the convergence of the laws it generates, in the limit as the number of extrapolation with fixed step size $\Delta t$ goes to infinity, to the invariant distribution of the underlying Euler-Maruyama scheme. When $\delta t$ denotes the microscopic step, the invariant distribution of the Euler-Maruyama scheme is the zero-mean normal distribution $\mathcal{N}_{0, V_{\infty}^{\delta t}}$, with variance

$$
V_{\infty}^{\delta t}=\delta t \sum_{j=0}^{\infty}(I+\delta t A)^{j} B B^{\top}\left(I+\delta t A^{\top}\right)^{j},
$$

as can be seen by repeatedly applying the recursion for the mean and variance in (7).

The stability question was analyzed in [15] in the Gaussian setting, where the distributions that are generated by the micro-macro acceleration can be computed explicitly. The main result there focuses on a simple diagonal case and reads:

Theorem. When applying the micro-macro acceleration method to the linear SDE (1) with blockdiagonal drift matrix

$$
A=\left[\begin{array}{cc}
A^{s} & 0 \\
0 & A^{f}
\end{array}\right]
$$


the mean $\mu_{n}$ and the covariance matrix $\Sigma_{n}$ of the resulting Gaussian law at the nth step satisfy

$$
\lim _{n \rightarrow \infty} \mu_{n}=0, \quad \lim _{n \rightarrow \infty} \Sigma_{n}=V_{\infty}^{\delta t}
$$

whenever

$$
\rho\left(I^{s}+\Delta t A^{s}\right)<1 \quad \text { and } \quad \rho\left(I^{s}+\delta t A^{f}\right)<1 .
$$

Here, $\rho(\cdot)$ denotes the spectral radius of a matrix. Condition (13) is necessary to stabilize the extrapolation of the slow mean $\mu_{n}^{s}$, by bounding the values of $\Delta t$, and to stabilize the EulerMaruyama stage, by bounding the values of $\delta t$. For the proof of Theorem 4 it suffices to establish the proper asymptotic behaviour of the means $\mu_{n}$ and variances $\Sigma_{n}$ of Gaussian distributions generated by the micro-macro acceleration scheme.

In this section, we go beyond the Gaussian case and work within the larger class of probability measures that have Gaussian tails. As a consequence, we do not have explicit formulas for the distributions generated by the scheme and we have to control all moments to show stability. Rather than trying to obtain stability bounds on $\Delta t$ directly, we concentrate on showing that convergence of $\mu^{n}$ to 0 , i.e., asymptotic stability of the mean, already yields convergence of the distributions to $\mathcal{N}_{0, V_{\infty}^{\delta t}}$. The relation between these two notions was illuminated in the Gaussian setting in [15].

The main stability result of this paper, Theorem 2 in Section 4.2 , gives weak convergence of distributions produced by the micro-macro acceleration method to $\mathcal{N}_{0, V_{\infty}^{\delta t}}$, as the number of extrapolation steps goes to infinity. To prove it, we explore the properties of cumulant generating functions (CGFs) to produce a recursion formula for the laws generated by the method (Section 4.1).

\subsection{Micro-macro step in terms of the cumulant generating function}

Let us first define the cumulant generating function as follows.

Definition 1. For any probability distribution $P \in \mathscr{P}^{d}$, we define the cumulant generating function of $P$

$$
\mathcal{K}_{P}(\theta)=\ln \mathbb{E}_{P}\left[e^{\theta \cdot \Pi}\right], \quad \theta \in \Theta_{P}
$$

where $\Pi$ is the identity on $\mathbb{R}^{d}$, and the effective domain reads $\Theta_{P}=\left\{\theta \in \mathbb{R}^{d}: \mathcal{K}_{P}(\theta)<+\infty\right\}$.

When $X \sim P$ we also write $\mathcal{K}_{X}$ instead of $\mathcal{K}_{P}$, and $\Theta_{X}$ instead of $\Theta_{P}$. We summarize the basic properties of CGFs in AppendixA.

Example 1. If $X \sim \mathcal{N}_{\mu, \Sigma}$, then

$$
\mathcal{K}_{X}(\theta)=\mu \cdot \theta+\frac{1}{2} \theta^{\top} \Sigma \theta .
$$

To effectively use the CGFs to describe the micro-macro acceleration procedure, we assume that for the initial random variable $X_{0}$ it holds $\Theta_{0} \doteq \Theta_{X_{0}}=\mathbb{R}^{d}$. In view of Proposition 6 , the CGF of the matched distribution results from shifting and translating the CGF of the prior based on the current value of the Lagrange multipliers. Having priors with full effective domain avoids the issue of falling outside the effective domain while shifting the CGF - a clear sign that the matching is impossible.

Remark 3 (What does $\Theta_{0}=\mathbb{R}^{d}$ mean?). Let us fix $\theta \in \mathbb{R}^{d}$ and $r>0$. According to the Chernoff's bound [24, p. 392], we have

$$
\mathbb{P}\left(\theta \cdot X_{0} \geq r\right) \leq e^{-s r+\mathcal{K}_{\theta} \cdot X_{0}(s)},
$$

for all $s \geq 0$. From Proposition 5 iii in the Appendix, applied with $l=1$ and $M=\theta^{\top}$, we have $\mathcal{K}_{\theta \cdot X_{0}}(s)=\mathcal{K}_{X_{0}}(s \theta)$. Thus, taking logarithms on both sides of 14 , we can equivalently write

$$
-\ln \mathbb{P}\left(\theta \cdot X_{0} \geq r\right) \geq s r-\mathcal{K}_{0}(s \theta),
$$


where we denote $\mathcal{K}_{0}=\mathcal{K}_{X_{0}}$. Since $\mathcal{K}_{0}(s \theta)$ is finite for all $s \geq 0$, dividing by $r$ and taking the limit gives

$$
\lim _{r \rightarrow+\infty} \frac{-\ln \mathbb{P}\left(\theta \cdot X_{0} \geq r\right)}{r} \geq s .
$$

Therefore, because $s$ can be arbitrarily large, the $\log$-tail function of $\theta \cdot X_{0}$ is superlinear at $+\infty$. The same holds at $-\infty$ by repeating the argument for $\mathbb{P}\left(\theta \cdot X_{0} \leq-r\right)$.

To simplify the notation, we again use only one micro step for each extrapolation in the micromacro acceleration procedure. In the Proposition below, we consider the micro-macro acceleration method as applied to the linear slow-fast SDE (1).

Proposition 3. Let $n \geq 1$ and assume that for a random variable $X_{n-1}$ with cumulant generating function $\mathcal{K}_{X_{n-1}}=\mathcal{K}_{n-1}$ we have $\Theta_{n-1}=\mathbb{R}^{d}$. Then, if $X_{n}$ is obtained from the micro-macro procedure with extrapolation of the (slow) s-marginal mean (as described in Section 2.2. with $K=$ 1), its CGF $\mathcal{K}_{n}=\mathcal{K}_{X_{n}}$ has effective domain $\Theta_{n}=\mathbb{R}^{d}$ and satisfies

$$
\begin{aligned}
\mathcal{K}_{n}(\theta)= & \mathcal{K}_{n-1}\left(\left(I+\delta t A^{\top}\right)\left(\theta+\lambda_{n}^{s} \oplus 0^{f}\right)\right)-\mathcal{K}_{n-1}\left(\left(I+\delta t A^{\top}\right)\left(\lambda_{n}^{s} \oplus 0^{f}\right)\right) \\
& +\frac{\delta t}{2}\left[\theta^{\top} B B^{\top} \theta+\left(\lambda_{n}^{s} \oplus 0^{f}\right)^{\top} B B^{\top} \theta+\theta^{\top} B B^{\top}\left(\lambda_{n}^{s} \oplus 0^{f}\right)\right],
\end{aligned}
$$

where $\lambda_{n}^{s}$ is a vector of Lagrange multipliers.

Proof. According to Section 2, the law of $X_{n}$ is given by $\mathcal{M}\left(\mu_{n}^{s}, \operatorname{Law}\left(X_{n-1,1}^{\delta t}\right)\right)$, where $\mu_{n}^{s}$ is extrapolated as in (6) and $X_{n-1,1}^{\delta t}$ is obtained from $X_{n-1}$ by one Euler-Maruyama step over $\delta t$. Therefore, for the cumulant, Proposition 6 yields

$$
\mathcal{K}_{n}(\theta)=\mathcal{K}_{n-1,1}\left(\theta+\lambda_{n}^{s} \oplus 0^{f}\right)-\mathcal{K}_{n-1,1}\left(\lambda_{n}^{s} \oplus 0^{f}\right),
$$

where $\lambda_{n}^{s}$ is the Lagrange multiplier associated to the extrapolated marginal mean $\mu_{n}^{s}$.

In the current notation, the recursive formula (5) reads

$$
X_{n-1,1}^{\delta t}=(I+\delta t A) X_{n-1}+B \delta W_{n, 1} .
$$

Because $X_{n-1}$ and $\delta W_{n, 1}$ are independent, we obtain from Proposition 5 v v that

$$
\mathcal{K}_{n-1,1}=\mathcal{K}_{(I+\delta t A) X_{n-1}}+\mathcal{K}_{B \delta W_{n, 1}} .
$$

The law of $B \delta W_{n, 1}$ is $\mathcal{N}_{0, \delta t B B^{\top}}$ thus, according to Example 1. $\mathcal{K}_{B \delta W_{n, 1}}(\theta)=\delta t / 2 \theta^{\top} B B^{\top} \theta$ and, using Proposition 5 (iii), we obtain

$$
\mathcal{K}_{n-1,1}(\theta)=\mathcal{K}_{n-1}\left(\left(I+\delta t A^{\top}\right) \theta\right)+\frac{\delta t}{2} \theta^{\top} B B^{\top} \theta .
$$

Combining (16) with 17) results in 15). Since the effective domain of $X_{n-1}$ was equal to $\mathbb{R}^{d}$, the right-hand side of 15 is finite for all $\theta$. This implies $\Theta_{n}=\mathbb{R}^{d}$.

\subsection{Convergence to the equilibrium with stable mean extrapolation}

The main result of this section depends on the following assumption on the initial random variable for the micro-macro acceleration method. Recall from Proposition 5 (iv) that the cumulant generating function is always analytic on the interior of its effective domain. Here and in what follows, for any constant $C \geq 0$ and functions $f, g: \mathbb{R}_{+} \rightarrow \mathbb{R}$ with $g$ positive in a neighbourhood of infinity, $f(r) \sim C g(r)$ means $\lim _{r \rightarrow+\infty} f(r) / g(r)=C$.

Assumption 1. The CGF $\mathcal{K}_{0}$, of an initial random variable $X_{0}$, has full effective domain (i.e., $\Theta_{0}=\mathbb{R}^{d}$ ) and for every $\theta \in \mathbb{R}^{d}$ it satisfies

$$
\mathcal{K}_{0}(r \theta) \sim w_{0}(\theta) r^{2}, \quad \frac{\mathrm{d}}{\mathrm{d} r} \mathcal{K}_{0}(r \theta) \sim 2 w_{0}(\theta) r
$$

where $w_{0}: \mathbb{R}^{d} \rightarrow(0,+\infty)$ is continuous and homogeneous of order 2 . 
Assumption 1 derives from the theory of regular variation. Let us first discuss its connection with the tails of random variables.

Remark 4 (On log-quadratic tails). The asymptotic relation $\mathcal{K}_{0}(r \theta) \sim w_{0}(\theta) r^{2}$ and Proposition 5 (iii) leads to $\mathcal{K}_{\theta \cdot X_{0}}(r) \sim w_{0}(\theta) r^{2}$. By the Kasahara-Tauberian Theorem [25, Thm. 4.12.7], the last relation is equivalent to the property

$$
-\ln \mathbb{P}\left(\theta \cdot X_{0} \geq r\right) \sim-\ln \mathbb{P}\left(\theta \cdot X_{0} \leq-r\right) \sim w_{0}(\theta)^{-1} r^{2}, \quad \text { as } r \rightarrow+\infty .
$$

That is, for every $\theta \in \mathbb{R}^{d}$, the random variable $\theta \cdot X_{0}$ has regularly varying log-quadratic tail decay, a feature shared by all Gaussian laws. Therefore, Assumption 1 sharpens the superlinear behaviour of tail functions that resulted from assuming $\Theta_{0}=\mathbb{R}^{d}$, see Remark 3 . The inclusion of derivatives in $[18$ is related to the notion of higher-order regular variation(compare [26] and [25, p. 44]).

In the proof of Theorem 2 below, we employ the following technical result.

Lemma 2. Let $\mathcal{K}$ be continuously differentiable function on $\mathbb{R}^{d}$ that for every $\theta \in \mathbb{R}^{d}$ satisfies $\mathcal{K}(r \theta) \sim w(\theta) r^{2}$ and

$$
\sup _{\theta \neq 0} \frac{\|\nabla \mathcal{K}(\theta)\|}{\|\theta\|}<+\infty .
$$

Then, for every $\theta, \theta_{0} \in \mathbb{R}^{d}$, it holds $\mathcal{K}\left(r \theta+\theta_{0}\right) \sim 2 w(\theta) r^{2}$.

Proof. Express the ratio $\mathcal{K}\left(r \theta+\theta_{0}\right) / r^{2}$ as the following sum

$$
\frac{\mathcal{K}\left(r \theta+\theta_{0}\right)-\mathcal{K}(r \theta)}{r^{2}}+\frac{\mathcal{K}(r \theta)}{r^{2}} .
$$

The second summand converges to $2 w(\theta)$ as $r \rightarrow+\infty$ from the asymptotic property of $\mathcal{K}$. That the first fraction disappears can be seen by applying the mean value inequality

$$
\frac{\left|\mathcal{K}\left(r \theta+\theta_{0}\right)-\mathcal{K}(r \theta)\right|}{r^{2}} \leq \frac{\left\|\nabla \mathcal{K}\left(r \theta+r^{\prime} \theta_{0}\right)\right\|\left\|\theta_{0}\right\|}{r^{2}}
$$

where $r^{\prime} \in[0,1]$. Since

$$
\frac{\left\|\nabla \mathcal{K}\left(r \theta+r^{\prime} \theta_{0}\right)\right\|}{r}=\frac{\left\|\nabla \mathcal{K}\left(r \theta+r^{\prime} \theta_{0}\right)\right\|}{\left\|r \theta+r^{\prime} \theta_{0}\right\|} \cdot\left\|\theta+\frac{r^{\prime}}{r} \theta_{0}\right\|
$$

stays bounded as $r \rightarrow+\infty$, due to $[19$, the right-hand side of the previous inequality converges to zero in this limit.

We are now ready to formulate and prove our stability result. In Theorem 2, we consider a class of initial distributions specified by the tail properties of their CGF $\mathcal{K}_{0}$. This class is chosen to extend on normal distributions and it incorporates, for example, the class of Gaussian mixtures. Many distributions appearing in multi-scale models can have a very complex, multi-modal shape inside bounded regions of the state space, but ultimately decay with Gaussian tails. The assumptions we make on $\mathcal{K}_{0}$ in Theorem 2 are insensitive to the complexity of initial conditions in any bounded domain and thus can be satisfied in plenty of problems.

Theorem 2. Suppose that the $C G F \mathcal{K}_{0}$ of an initial random variable $X_{0}$ satisfies Assumption 1 , both $\mathcal{K}_{0}(\theta)$, and $\nabla \mathcal{K}_{0}(\theta) \cdot \theta$ fulfill $\left[19\right.$, and $\mathcal{K}_{0}$ has all derivatives of order 3 or higher bounded. If $\rho(I+\delta t A)<1$, and the mean $\mu_{n}$ of random variables $X_{n}$ obtained from the micro-macro acceleration method is stable, that is $\lim _{n \rightarrow+\infty} \mu_{n}=0$, the laws of $X_{n}$ converge weakly to $\mathcal{N}_{0, V_{\infty}^{\delta t}}$.

The requirement that $\rho(I+\delta t A)<1$ ensures the stability of underlying Euler-Maruyama scheme for microscopic integration. In practice, we need to choose microscopic time step $\delta t$ small enough so that $|1+\delta t \lambda|<1$ for all eigenvalues $\lambda$ of the drift matrix $A$, see also Remark 1. The stability of 
the mean $\mu_{n}$ is closely related to the stability bounds on $\Delta t$ that guarantee that the slow marginal mean $\mu_{n}^{s}$ satisfies $\lim _{n \rightarrow \infty} \mu_{n}^{s}=0$. The behaviour of fast marginal $\mu_{n}^{f}$ is influenced at each step by both extrapolation over $\Delta t$ and the matching. In the Gaussian setting, Lemma 1 provides the exact formula for $\mu_{n}^{f}$ in terms of $\mu_{n}^{s}$ and the first two moments of distributions produced by the Euler-Maruyama step. Using this formula, we can obtain explicit stability bounds on $\Delta t$ and $\delta t$, exemplified in (13). In the non-Gaussian setting, we can only say that the influence of matching on $\mu_{n}^{f}$ is encoded in the nonlinear procedure to obtain Lagrange multipliers $\lambda_{n}$. Having no explicit formulas for the multipliers, we work under the assumption that the full mean is stable when using the micro-macro acceleration method. This assumption is closely related to the stability of the extrapolation, which only requires the macro time step $\Delta t$ to be on the order of the largest eigenvalue of the slow drift $A^{s}$, see Remark 1. Because distributions propagated by the micromacro acceleration method are not described by first two moments any more, in contrast with Section 3 , in the proof we need to analyze the whole infinite sequence of cumulants.

Proof. To establish stability, we employ the recursive relation (15). However, due to the presence of the Lagrange multipliers $\lambda_{n}^{s}$ in the argument of $\mathcal{K}_{n}$ and in the additional last term of (15), we cannot immediately pass to the limit as $n$ goes to $+\infty$. These multipliers exist for all $n$, a consequence of $\Theta_{n}=\mathbb{R}^{d}$, but we do not have any a priori estimates that would allow to control $\lambda_{n}^{s}$ as $n$ increases. Therefore, our strategy in proving the convergence is to look at the recurrences for the tails of $\mathcal{K}_{n}$, which do not contain $\lambda_{n}^{s}$ any more. Before we look at $\mathcal{K}_{n}$ itself, let us use the boundedness of its higher order derivatives to show the convergence of higher cumulants.

First note that the last term in formula (15) is of second order in $\theta$, so it disappears after differentiating this identity three times. More precisely, we have, for any $j \geq 3$,

$$
\mathrm{D}^{j} \mathcal{K}_{n}(\theta)\left[\theta^{\prime}\right]=\mathrm{D}^{j} \mathcal{K}_{n-1}\left(\left(I+\delta t A^{\top}\right)\left(\theta+\lambda_{n}^{s} \oplus 0^{f}\right)\right)\left[\left(I+\delta t A^{\top}\right) \theta^{\prime}\right]
$$

where we treat the $j$ th order derivative $\mathrm{D}^{j} \mathcal{K}_{n}(\theta)$ at $\theta \in \mathbb{R}^{d}$ as a symmetric multilinear mapping on $\left(\mathbb{R}^{d}\right)^{j}$ and denote $\mathrm{D}^{j} \mathcal{K}_{n}(\theta)\left[\theta^{\prime}\right] \doteq \mathrm{D}^{j} \mathcal{K}_{n}(\theta)\left[\theta^{\prime}, \ldots, \theta^{\prime}\right]$. By back-substituting we obtain

$$
\mathrm{D}^{j} \mathcal{K}_{n}(\theta)\left[\theta^{\prime}\right]=\mathrm{D}^{j} \mathcal{K}_{0}\left(\left(I+\delta t A^{\top}\right)^{n} \theta+\sum_{k=1}^{n}\left(I+\delta t A^{\top}\right)^{k}\left(\lambda_{k}^{s} \oplus 0^{f}\right)\right)\left[\left(I+\delta t A^{\top}\right)^{n} \theta^{\prime}\right] .
$$

Since $\mathrm{D}^{j} \mathcal{K}_{0}$ is bounded on $\mathbb{R}^{d}$ for all $j \geq 3$, we can estimate from the above relation that

$$
\left\|\mathrm{D}^{j} \mathcal{K}_{n}(\theta)\right\|_{\text {mult }} \leq \max _{\theta^{\prime} \in \mathbb{R}^{d}}\left\|\mathrm{D}^{j} \mathcal{K}_{0}\left(\theta^{\prime}\right)\right\|_{\text {mult }}\left\|I+\delta t A^{\top}\right\|^{j n}
$$

where $\left\|\mathrm{D}^{j} \mathcal{K}_{n}(\theta)\right\|_{\text {mult }} \doteq \sup \left|\mathrm{D}^{j} \mathcal{K}_{n}(\theta)\left(\theta^{1}, \ldots, \theta^{j}\right)\right| /\left\|\theta^{1}\right\| \cdots\left\|\theta^{j}\right\|$ and the supremum is taken over all $\theta^{i} \neq 0$. Since $\rho(I+\delta t A)<1$, this demonstrates that $\mathrm{D}^{j} \mathcal{K}_{n}(\theta)$ converges to zero as $n \rightarrow+\infty$, uniformly in $\theta$. In particular, $\lim _{n \rightarrow+\infty} \mathrm{D}^{j} \mathcal{K}_{n}(0)=0$ for all $j \geq 3$.

Let us now return to 15 . We show by induction that for all $\theta \in \mathbb{R}^{d}$ the function $r \mapsto \mathcal{K}_{n}(r \theta)$ is asymptotically quadratic in $r$ and find the recursion for the corresponding constants $w_{n}(\theta)$. Suppose that $\mathcal{K}_{n-1}(r \theta) \sim w_{n-1}(\theta) r^{2}$ with some function $w_{n-1}$. Note that Assumption 1 guarantees that this holds for $\mathcal{K}_{0}$. The recursive relation 15 gives

$$
\begin{aligned}
\frac{\mathcal{K}_{n}(r \theta)}{r^{2}}= & \frac{\mathcal{K}_{n-1}\left(r\left(I+\delta t A^{\top}\right) \theta+\left(I+\delta t A^{\top}\right)\left(\lambda_{n}^{s} \oplus 0^{f}\right)\right)}{r^{2}}-\frac{\mathcal{K}_{n-1}\left(\left(I+\delta t A^{\top}\right)\left(\lambda_{n}^{n} \oplus 0^{f}\right)\right.}{r^{2}} \\
& +\frac{\delta t}{2} \frac{r^{2} \theta^{\top} B B^{\top} \theta+r\left(\lambda_{n}^{s} \oplus 0^{f}\right)^{\top} B B^{\top} \theta+r \theta^{\top} B B^{\top}\left(\lambda_{n}^{s} \oplus 0\right)}{r^{2}}
\end{aligned}
$$

Note that simultaneously, by differentiating $(15)$, the gradient of $\mathcal{K}_{n}$ satisfies

$$
\nabla \mathcal{K}_{n}(\theta)=(I+\delta t A) \nabla \mathcal{K}_{n-1}\left(\left(I+\delta t A^{\top}\right)\left(\theta+\lambda_{n}^{s} \oplus 0^{f}\right)\right)+\delta t\left(\theta+\lambda_{n}^{s} \oplus 0^{f}\right)^{\top} B B^{\top}
$$


thus property (19) propagates from $\mathcal{K}_{0}$ throughout all $\mathcal{K}_{n}$. Therefore, we can use Lemma 2 together with the inductive assumption, to conclude that the limit as $r$ goes to $+\infty$ on the right hand side exists and is equal to $2 w_{n-1}\left(\left(I+\delta t A^{\top}\right) \theta\right)+\delta t \theta^{\top} B B^{\top} \theta$. Denoting by $2 w_{n}(\theta)$ the limit of the left hand side, we have the recursion $w_{n}(\theta)=w_{n-1}\left(\left(I+\delta t A^{\top}\right) \theta\right)+(\delta t / 2) \theta^{\top} B B^{\top} \theta$. By back-substituting, we obtain

$$
w_{n}(\theta)=w\left(\left(I+\delta t A^{\top}\right)^{n} \theta\right)+\frac{\delta t}{2} \theta^{\top}\left[\sum_{j=0}^{n-1}(I+\delta t A)^{j} B B^{\top}\left(I+\delta t A^{\top}\right)^{j}\right] \theta .
$$

Note also that, by a similar reasoning as for $\mathcal{K}_{n}$, but using (21) this time, we can inductively establish the relation $\mathrm{d} / \mathrm{d} r \mathcal{K}_{n}(r \theta) \sim 2 w_{n}(\theta) r$. In particular, after differentiating, this relation yields

$$
\lim _{r \rightarrow+\infty} \frac{\nabla \mathcal{K}_{n}(r \theta) \cdot \theta}{r}=2 w_{n}(\theta),
$$

with $w_{n}$ satisfying 22 .

Since the micro time step $\delta t$ is stable, we can now take the limit as $n \rightarrow \infty$ on the right-hand side of 222. In consequence, we know that the point-wise limit $\omega_{\infty} \doteq \lim _{n \rightarrow \infty} \omega_{n}$ exists and, by 12. , results in the following expression for the limiting function

$$
w_{\infty}(\theta)=\frac{1}{2} \theta^{\top}\left[\delta t \sum_{j=0}^{\infty}(I+\delta t A)^{j} B B^{\top}\left(I+\delta t A^{\top}\right)^{j}\right] \theta=\frac{1}{2} \theta^{\top} V_{\infty}^{\delta t} \theta .
$$

Having established the limiting behaviour of higher cumulants $\mathrm{D}^{j} \mathcal{K}_{n}(0)$, for $j \geq 3$, we will now use identity 24) to demonstrate that the second cumulants $\nabla^{2} \mathcal{K}_{n}(0)$ converge to $V_{\infty}^{\delta t}$. Via the fundamental theorem of calculus we have

$$
\int_{0}^{1} r \theta^{\top} \nabla^{2} \mathcal{K}_{n}(\operatorname{tr} \theta) \theta \mathrm{d} t=\nabla \mathcal{K}_{n}(r \theta) \cdot \theta-\nabla \mathcal{K}_{n}(0) \cdot \theta .
$$

Dividing by $r$ and changing variable of integration $t \mapsto t / r$ gives

$$
\lim _{r \rightarrow+\infty} \frac{1}{r} \int_{0}^{r} \theta^{\top} \nabla^{2} \mathcal{K}_{n}(t \theta) \theta \mathrm{d} t=\lim _{r \rightarrow+\infty}\left(\frac{\nabla \mathcal{K}_{n}(r \theta) \cdot \theta}{r}-\frac{\nabla \mathcal{K}_{n}(0) \cdot \theta}{r}\right)=2 w_{n}(\theta),
$$

by the asymptotic property (23) of $r \mapsto \nabla \mathcal{K}_{n}(r \theta) \cdot \theta$. Denoting $f_{0}^{\infty} \doteq \lim _{r \rightarrow+\infty} \frac{1}{r} \int_{0}^{r}$, we have from 24

$$
f_{0}^{\infty} \theta^{\top} \nabla^{2} \mathcal{K}_{n}(t \theta) \theta \mathrm{d} t=2 w_{\infty}(\theta)=\theta^{\top} V_{\infty}^{\delta t} \theta .
$$

Because the Taylor expansion of $t \mapsto \mathcal{K}_{n}(t \theta)$ around $t=0$ gives $\nabla^{2} \mathcal{K}_{n}(t \theta)=\nabla^{2} \mathcal{K}_{n}(0)+\mathrm{D}^{3} \mathcal{K}_{n}\left(\theta_{t}^{\prime}\right)$, with some $\theta_{t}^{\prime}$, and $\mathrm{D}^{3} \mathcal{K}_{n}\left(\theta_{t}^{\prime}\right)$ converges to zero as $n$ goes to $+\infty$, uniformly in $\theta_{t}^{\prime}$, we get

$$
\lim _{n \rightarrow+\infty} \theta^{\top} \nabla^{2} \mathcal{K}_{n}(0) \theta=\lim _{n \rightarrow+\infty} f_{0}^{\infty} \theta^{\top} \nabla^{2} \mathcal{K}_{n}(t \theta) \theta \mathrm{d} t=\theta^{\top} V_{\infty}^{\delta t} \theta,
$$

for any $\theta \in \mathbb{R}^{d}$. This proves the convergence of the second cumulants.

In conclusion, since $\nabla \mathcal{K}_{n}(0)=\mu_{n}$ converges to 0 , by assumption, $\nabla^{2} \mathcal{K}_{n}(0)$ converges to $V_{\infty}^{\delta t}$, and all higher order derivatives to 0 , we obtain the following limiting sequence of cumulants as $n$ goes to $+\infty$ :

$$
\left(0, V_{\infty}^{\delta t}, 0, \ldots\right) .
$$

This sequence uniquely determines the normal distribution with mean zero and covariance matrix $V_{\infty}^{\delta t}$. Thus, from the Frechét-Shohat theorem [27, p. 307], the laws of $X_{n}$ converge weakly to $\mathcal{N}_{0, V_{\infty}^{\delta t}}$. 


\section{Numerical illustrations}

In this section, we present two numerical illustrations regarding the micro-macro acceleration method. First, in Section 5.1 we perform numerical experiments showing the convergence and the stability region on a linear SDE with additive noise. Second, in Section 5.2 we compare the efficiency of micro-macro acceleration to other acceleration methods on a non-linear test case where the slow dynamics is linear and where the fast component has a bimodal dynamics.

\subsection{A periodically driven linear slow-fast system}

In this section, we numerically illustrate the convergence and stability results of the previous sections on an academic example. As in [5, 16], we define a linear slow-fast SDE with additive noise, in which we add a periodic forcing to the slow component

$$
\left\{\begin{array}{l}
d X=-2(X+Y) d t+\sin (2 \pi t) d t+d W_{x} \\
d Y=\frac{1}{\varepsilon}(X-Y) d t+\frac{1}{\sqrt{\varepsilon}} d W_{y} .
\end{array}\right.
$$

The parameter $\varepsilon$ is the time-scale separation between $X$ and $Y$ and controls the stiffness in the system. Introducing a periodic forcing allows to easily measure errors between the exact and numerical solution by computing the $L^{2}$ difference between both curves over one period. At the same time, the driving force does not alter stability and convergence properties of micro-macro acceleration.

We first look at some numerical convergence tests (Section 5.1.1), before moving on to computational experiments on stability (Section 5.1.2.

\subsubsection{Convergence to the microscopic time integrator}

By taking expectations of (26), one can show that the means $\mu_{X}$ (resp. $\mu_{Y}$ ) of the slow (resp. fast) component of the exact solution are given by

$$
\left(\begin{array}{l}
\mu_{X}(t) \\
\mu_{Y}(t)
\end{array}\right)=e^{t M}\left(\begin{array}{l}
\mu_{X_{0}}-A \\
\mu_{Y_{0}}-C
\end{array}\right)+\left(\begin{array}{l}
A \\
C
\end{array}\right) \cos (2 \pi t)+\left(\begin{array}{l}
B \\
D
\end{array}\right) \sin (2 \pi t)
$$

where $M=\left(\begin{array}{cc}-2 & -2 \\ 10 & -10\end{array}\right)$ and $\left(\begin{array}{ll}\mu_{X_{0}} & \mu_{Y_{0}}\end{array}\right)^{T}$ is the mean of the initial condition of 26). The constants $A, B, C$ and $D$ are the solution of the linear system

$$
\left(\begin{array}{cccc}
-2 \pi & 2 & 0 & 2 \\
2 & 2 \pi & 2 & 0 \\
0 & -10 & -2 \pi & 10 \\
-10 & 0 & 10 & 2 \pi
\end{array}\right)\left(\begin{array}{l}
A \\
B \\
C \\
D
\end{array}\right)=\left(\begin{array}{l}
1 \\
0 \\
0 \\
0
\end{array}\right)
$$

To illustrate convergence (Theorem 1), we compute the error in the slow means obtained micromacro acceleration against both the exact solution (27) and the numerical result obtained by the Euler-Maruyama integrator. We are mostly interested in the error between micro-macro acceleration and the Euler-Maruyama method because we want to understand the effect of extrapolation. As parameters we take $\varepsilon=0.5$ and $\varepsilon=0.05$ for the time-scale separation and we perform computations to an end time $T=6$. We choose a small time step $\delta t=\varepsilon / 20$ for the microscopic time integrator and many values for the extrapolation time step $\Delta t$. The error is computed as the $L_{2}$ norm of the difference between two curves and averaged over 10 independent runs. The convergence results are depicted on Figure 1 .

Figure 1 shows that the micro-macro acceleration error lowers as $\Delta t$ decreases to 0 , as proven in Theorem 1 For a large $\varepsilon=0.5$ the error decreases linearly, while for a small $\varepsilon=0.05$ the decrease 

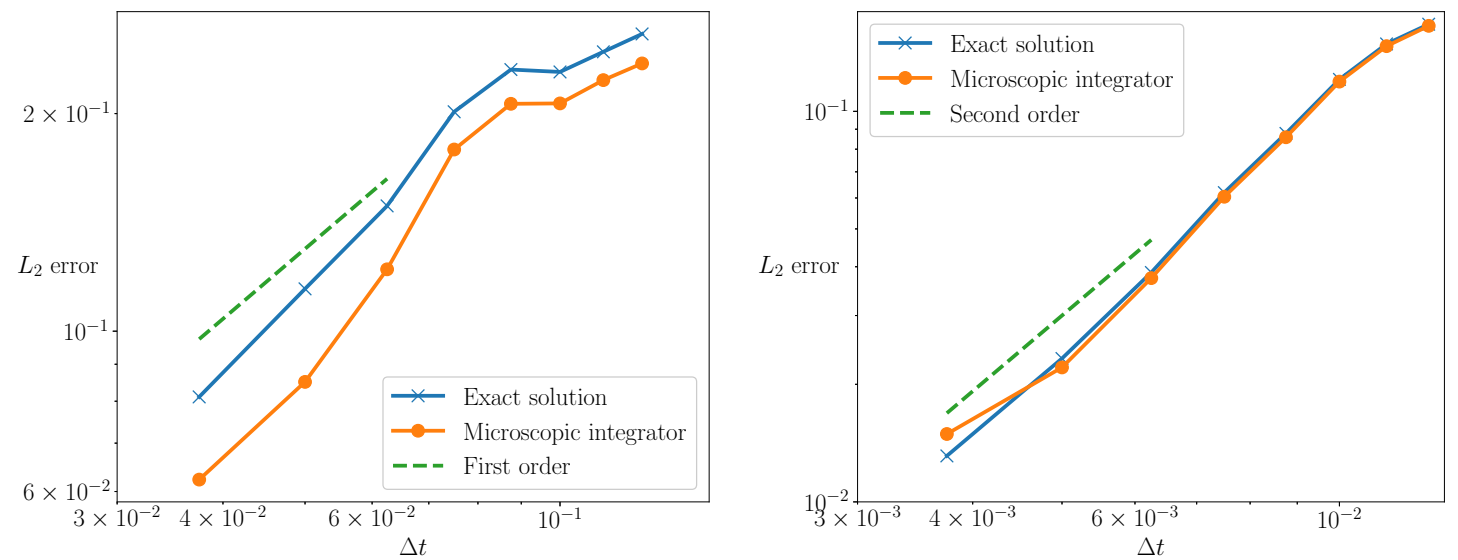

Figure 1: The error in the slow mean of micro-macro acceleration as a function of the extrapolation step size $\Delta t$, computed against the analytic solution 27p (blue) and against the numerical result obtained by the Euler-Maruyama method with time step $\delta t$ (orange) for $\varepsilon=0.5$ (left) and $\varepsilon=0.05$ (right). We clearly see that the micro-macro acceleration error decreases when $\Delta t$ decreases, as given by Theorem 1 . Moreover, for small $\varepsilon$ there is almost no difference between the error computed against the analytic solution and the microscopic time integrator as the latter is very accurate. This difference is higher for larger $\varepsilon$.

is quadratically. The order of convergence of micro-macro acceleration is not well-understood yet. In Figure 1 we also see that the error computed against the analytic solution and the EulerMaruyama method is almost the same for small $\varepsilon=0.05$ since we use small time steps for the Euler-Maruyama time-integrator. For larger $\varepsilon$, the error between micro-macro acceleration and the Euler-Maruyama method is smaller than with the exact solution because the Euler-Maruyama method makes a non-negligible error.

\subsubsection{Stability with initial conditions having Gaussian tails}

In the next experiment, we also consider the periodically driven linear system, but look at large extrapolation steps to study stability properties. The derivation in Section 4 does not give a threshold on the extrapolation step above which micro-macro acceleration becomes unstable and below which the algorithm is stable. To determine that a simulation was unstable, we will therefore rely on an alternative strategy, also proposed in [15 for Gaussian initial conditions. In [15, it was shown that instability of the micro-macro acceleration technique unavoidably leads to so-called matching failures, even before the solution blows up to infinity. A matching failure occurs when there exists no probability distribution that is consistent with the given macroscopic state variables $m$. In other words, the pair $(m, \mu)$ does not lie in the domain of the matching operator $\mathcal{M}$ for any prior distribution $\mu$, unless $\mu$ is consistent with $m$.

In practice, we employ a Newton-Raphson method to compute the Lagrange multipliers in (3) and detect a matching failure when the iterative method does not converge. Specifically, we must solve

$$
\int_{G} x \exp \left(\bar{\lambda}^{s} \cdot x-A\left(\bar{\lambda}^{s}, \mu\right)\right) d P^{s}(x)=\bar{\mu}^{s},
$$

with a Newton-Raphson procedure, where we compute the integral using a Monte-Carlo representation of the prior distribution $P$. When the Newton-Raphson solver fails to reach the extrapolated slow mean $\bar{\mu}^{s}$ within an absolute tolerance of $10^{-11}$ in 50 steps, we mark a matching failure.

For the numerical experiment, we simulate the periodically driven linear system (26) with $\epsilon=1$ for different pairs of step sizes $(\delta t, \Delta t)$ up to the end time $T=100$. When a matching failure occurs, we mark the pair of parameters as unstable, and stable otherwise. The initial condition is Gaussian with mean zero and unit variance, which fits in the framework of Section 4. The number of Monte 


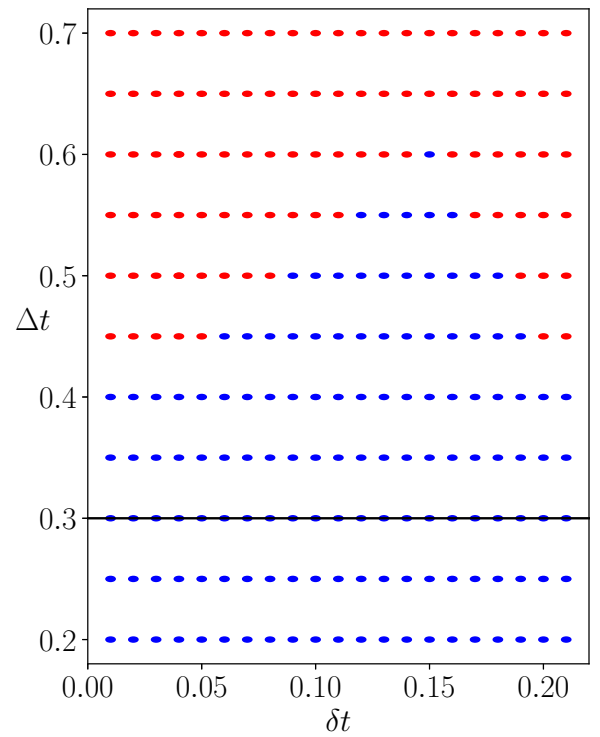

Figure 2: The $(\delta t, \Delta t)$ stability plane of micro-macro acceleration on the linear driven system 26. A blue dot indicates stability and a red dot instability. When at least one matching failure occurs, we mark an instability at the corresponding time step sizes. The stability domain has a V-shaped domain, and for every microscopic time step, the maximal extrapolation time step is larger than the deterministic stability bound of 0.3 .

Carlo replicas is $N=10^{5}$ and we use $K=1$ step in the microscopic time integrator. The numerical results are summarized in Figure 2.

Since $M$ is neither diagonal nor lower-triangular, there exist no stability bounds on the extrapolation step $\Delta t$ yet [15]. As a proxy, we first compute the deterministic stability bound of the corresponding system of ODEs given by the drift term in 267, as if there were no Brownian motion. The eigenvalues of $M$ are $-6 \pm 2 i$, implying the maximal deterministic time step is $\Delta t_{\max }=0.3$.

Numerically, the stability domain of micro-macro acceleration is V-shaped, and for every $\delta t$ the maximal extrapolation step before instability is always greater than 0.3. Hence, the stability bound of micro-macro acceleration is always greater than the stability boundary of the associated system of ODEs. Micro-macro acceleration hence has favorable stability properties.

\subsection{Numerical comparison of micro-macro acceleration to other acceleration methods}

To show the efficiency of micro-macro acceleration, we consider a non-linear slow-fast system with additive noise, given by the set of stochastic differential equations

$$
\left\{\begin{array}{l}
d X=-(2 X+Y) d t+0.1 d W_{x} \\
d Y=-\frac{1}{\varepsilon}\left(Y^{3}-Y\right) d t+\frac{1}{\sqrt{\varepsilon}} d W_{y} .
\end{array}\right.
$$

The fast component $Y$ has a bimodal invariant measure $\mu_{\infty}(y) \propto \exp \left(-\left(\frac{1}{4} y^{4}-\frac{1}{2} y^{2}\right)\right)$ and is autonomous. The slow component $X$, however, is linear but influenced by $Y$. In this numerical example, we are interested in the time-evolution of the mean and variance of $X(t)$. We therefore extrapolate the mean and the second moment of $X(t)$ in the micro-macro acceleration algorithm. For more details on micro-macro acceleration with more than one variable, as introduced in this manuscript, we refer to [13].

In the following numerical experiment, we compare the efficiency micro-macro acceleration to three other methods: a microscopic simulation with the Euler-Maruyama method, a homogenization method [10] and coarse projective integration with lifting [8, 9]. We first explain these three methods briefly in the following paragraphs before comparing the methods numerically. 
Microscopic simulation. For the microscopic simulation, we employ a small time step $\delta t=\varepsilon / 10$ as a proxy for the exact time-evolution of (28) since an analytic solution is not available. We assume the Euler-Maruyama is almost exact, since the numerical errors of the other three methods are larger than those of the microscopic integrator. We also use the Euler-Maruyama method with time step $\delta t=\varepsilon / 10$ for the microscopic simulation in micro-macro acceleration and coarse projective integration to make a fair comparison with the full microscopic simulation.

Homogenization method. When the time-scale separation between $X$ and $Y$ is large ( $\varepsilon$ small), one can derive an approximate macroscopic model for $X$ in the form of a closed SDE in $X$. This procedure is called homogenization [10]. The idea is to average $Y$ against its invariant distribution $\mu_{\infty}(y)$ and to substitute the obtained value into the evolution equation of $X$. For the model problem (28), we obtain that the average of $Y$ is 0 , so the approximate macroscopic model for $X$ is

$$
d X=-2 X d t+0.1 d W .
$$

When $\varepsilon$ decreases to zero, one can show that the true dynamics of $X(t)$ given by 28 converges weakly to the approximate macroscopic model. For non-zero $\varepsilon$, the approximate model makes a modeling error, however.

Coarse Projective Integration with lifting. As a final method, we consider the coarse projective integration method with lifting instead of matching. The algorithm is identical to the micro-macro acceleration method, but instead of matching, we perform a constrained simulation to obtain an equilibrium distribution that is consistent with the extrapolated moments. This constrained simulation process, starting from the prior distribution, is called lifting. For more information on the lifting step, we refer to [28. In the following numerical experiment, we use 30 constrained steps with size $\delta=\varepsilon / 10$.

Discussion of the numerical results. For the numerical experiment, we choose the time-scale separation as $\varepsilon=5 \cdot 10^{-3}$ and simulate the bimodal problem (28) up time $T=4$. For micro-macro acceleration, we use extrapolation steps of size $5 \delta t, 10 \delta t$ and $20 \delta t$. The number of constrained steps for the lifting procedure is 30 ..

On Figure 3 we plot the variance of $X$ as a function of time. The microscopic simulation with Euler-Maruyama is depicted in blue, the homogenization model in orange and coarse-projective integration in brown. The numerical results of micro-macro acceleration are shown in solid lines with extrapolation time steps $\Delta t=5 \delta t$ (green), $\Delta t=10 \delta t$ (red) and $\Delta t=20 \delta t$ (purple). Based on the numerical results on Figure 3 , we conclude that the homogenization method reaches a steadystate distribution that has a lower variance than the exact steady state distribution of the model problem 28. In this model problem, the fast component $Y(t)$ has an impact on the evolution of $X(t)$ and induces a natural variance on $X$ since $Y$ is stochastic as well. This extra variance is not accounted for in the homogenized model, which hence has a lower steady-state variance.

The micro-macro acceleration method follows the transient dynamics of $X(t)$ well and has almost the same steady state value for the variance as the Euler-Maruyama method, which we consider to be the exact. Moreover, the accuracy of micro-macro acceleration is almost the same for a big range of extrapolation step sizes, implying we can accelerate the Euler-Maruyama simulation without losing much accuracy. Finally, we also see that the coarse projective integration method gives a completely inaccurate transient for the same time steps as the micro-macro acceleration method. During lifting, the objective is to find an equilibrium distribution that is consistent with the extrapolated moments. In this numerical example, we assume we have such a distribution after 30 constrained steps. However, during matching, we compute the unique distribution that is closest to the prior distribution in Kullback-Leibler sense, while also being consistent with the 


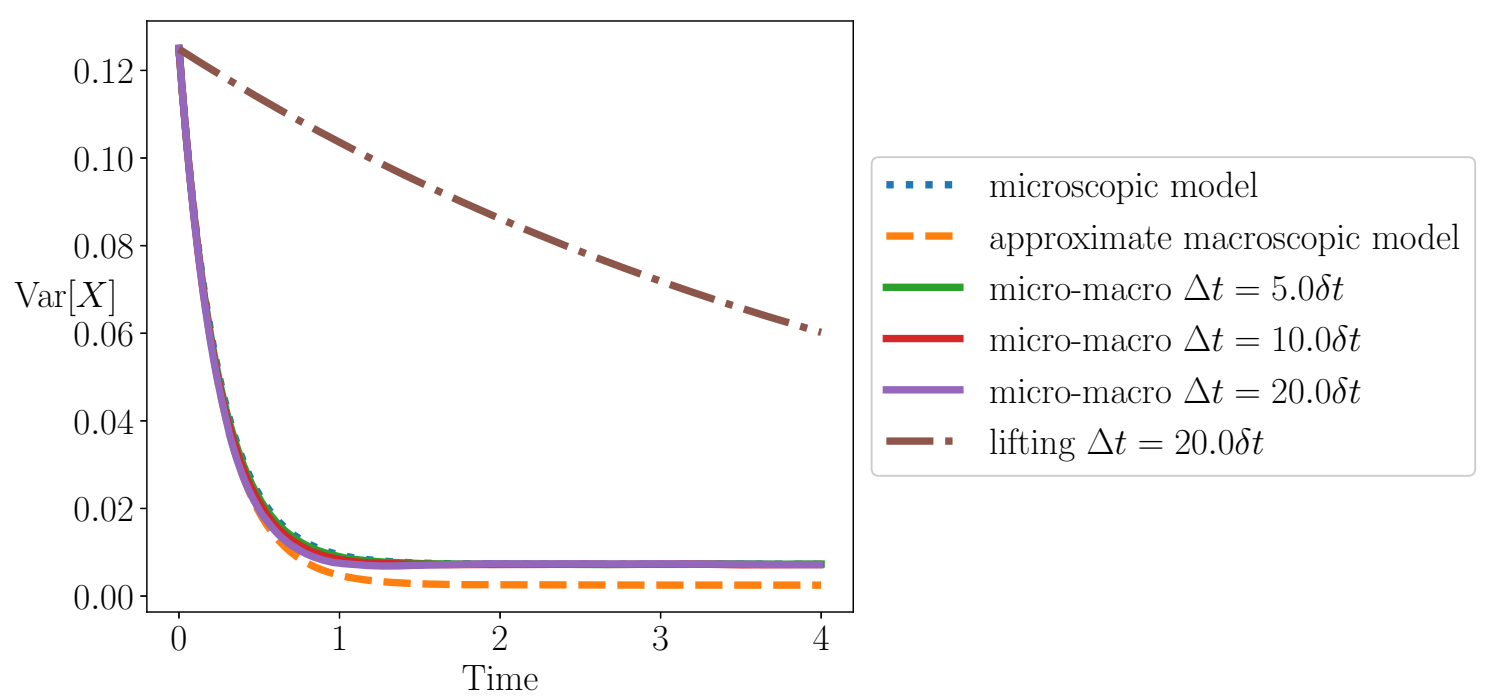

Figure 3: Time evolution of the variance of the slow mode $X$ 28, computed with the Euler-Maruyama method (blue dotted line), the homogenization method 29 (orange dashed line), coarse projective integration with lifting (brown dot-dashed line) and micro-macro acceleration (solid lines) with extrapolation time steps $5 \delta t$ (green), $10 \delta t$

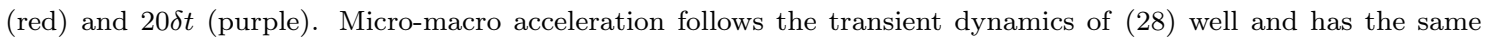
steady state value.

extrapolated moments. In lifting, there is no guarantee that the equilibrated distribution is the close to the prior. We can thus expect that matching makes no modeling error, yielding a much smaller error than lifting. On Figure 3 , the error made by lifting is indeed much larger than that of matching.

To conclude, micro-macro acceleration is able to take significantly larger time steps than the Euler-Maruyama method through extrapolation, while still obtaining a better accuracy than existing acceleration and homogenization methods.

\section{Conclusion}

We presented a micro-macro acceleration scheme, based on a combination of microscopic simulation and extrapolation of some macroscopic quantities of interest. We demonstrated that using only the slow mean during extrapolation results in a convergent and stable algorithm. The proofs hold for linear stochastic differential equations with additive noise. We complemented the analysis with numerical results that indicate that the error of micro-macro acceleration decreases to zero when both the extrapolation and microscopic step size decrease to zero. For stability, we investigated for which pairs microscopic and extrapolation time steps micro-macro acceleration is stable, and compared the numerical results to the deterministic stability bounds in the case without Brownian motion. Empirically, the stability domain is V-shaped, and for every value of the microscopic time step, the maximal extrapolation step is above the deterministic stability bound. We also compared numerically the efficiency of micro-macro acceleration to the Euler-Maruyama method, a homogenization scheme and a coarse projective integration method with lifting on a non-linear bimodal example. The micro-macro acceleration scheme is able to take significantly larger time steps than the inner Euler-Maruyama time integrator, while still obtaining a better accuracy than the homogenization and acceleration methods, proving efficiency of the micro-macro acceleration scheme. 


\section{Acknowledgements}

The authors thank Kristian Debrabant for proofreading the material in Section 4 The authors acknowledge the support of the Research Council of the University of Leuven through grant 'PDEOPT' and of the Research Foundation - Flanders (FWO - Vlaanderen) under grant G.A003.13.

\section{References}

[1] J. Fish, Bridging the scales in nano engineering and science, Journal of Nanoparticle Research 8 (5) (2006) 577-594.

[2] M. O. Steinhauser, Computational Multiscale Modeling of Fluids and Solids, Springer, 2017.

[3] Y. W. Kwon, D. H. Allen, R. Talreja, Multiscale modeling and simulation of composite materials and structures, Vol. 47, Springer, 2008.

[4] T. S. Deisboeck, G. S. Stamatakos, Multiscale cancer modeling, CRC Press, 2010.

[5] T. Li, A. Abdulle, W. E, Effectiveness of implicit methods for stiff stochastic differential equations, Communications in Computational Physics 3 (2) (2008) 295-307.

[6] W. E, B. Engquist, Multiscale modeling and computation, Notices of the AMS 50 (9) (2003) 1062-1070.

[7] A. Abdulle, W. E, B. Engquist, E. Vanden-Eijnden, The heterogeneous multiscale method, Acta Numerica 21 (2012) 1-87.

[8] I. G. Kevrekidis, C. W. Gear, J. M. Hyman, P. G. Kevrekidis, O. Runborg, C. Theodoropoulos, Equation-free, coarse-grained multiscale computation: Enabling mocroscopic simulators to perform system-level analysis, Communications in Mathematical Sciences 1 (4) (2003) 715762 .

[9] I. G. Kevrekidis, G. Samaey, Equation-free multiscale computation: Algorithms and applications, Annual review of physical chemistry 60 (2009) 321-344.

[10] G. Pavliotis, A. Stuart, Multiscale methods: averaging and homogenization, Springer Science \& Business Media, 2008.

[11] A. Abdulle, S. Cirilli, Stabilized methods for stiff stochastic systems, Comptes Rendus Mathematique 345 (10) (2007) 593-598.

[12] A. Abdulle, S. Cirilli, S-ROCK: Chebyshev methods for stiff stochastic differential equations, SIAM Journal on Scientific Computing 30 (2) (2008) 997-1014.

[13] K. Debrabant, G. Samaey, P. Zieliński, A micro-macro acceleration method for the Monte Carlo simulation of stochastic differential equations, SIAM Journal on Numerical Analysis 55 (6) (2017) 2745-2786.

[14] T. Lelièvre, G. Samaey, P. Zieliński, Analysis of a micro-macro acceleration method with minimum relative entropy moment matching, arXiv preprint arXiv:1801.01740.

[15] K. Debrabant, G. Samaey, P. Zieliński, Study of micro-macro acceleration schemes for linear slow-fast stochastic differential equations with additive noise, arXiv:1805.10219.

[16] H. Vandecasteele, P. Zieliński, G. Samaey, Efficiency of a micro-macro acceleration method for scale-separated stochastic differential equations, In preparation. 
[17] I. Karatzas, S. E. Shreve, Brownian Motion and Stochastic Calculus, 2nd Edition, Vol. 113 of Graduate Texts in Mathematics, Springer New York, 1998.

[18] R. M. Dudley, Real Analysis and Probability, Vol. 47, Cambridge University Press, 2002.

[19] M. Gasca, T. Sauer, Polynomial interpolation in several variables, Advances in Computational Mathematics 12 (4) (2000) 377.

[20] H. Schurz, Preservation of probabilistic laws through euler methods for ornstein-uhlenbeck process, Stochastic analysis and applications 17 (3) (1999) 463-486.

[21] C. R. Johnson, R. A. Horn, Matrix Analysis, Cambridge University Press, 1985.

[22] J. R. Hershey, P. A. Olsen, Approximating the kullback leibler divergence between gaussian mixture models, in: 2007 IEEE International Conference on Acoustics, Speech and Signal Processing - ICASSP '07, Vol. 4, 2007, pp. IV-317-IV-320. doi:10.1109/ICASSP.2007. 366913

[23] P. E. Kloeden, E. Platen, Numerical solution of stochastic differential equations, Vol. 23, Springer Science \& Business Media, 2013.

[24] T. M. Cover, J. A. Thomas, Elements of information theory, John Wiley \& Sons, 2012.

[25] N. H. Bingham, C. M. Goldie, J. L. Teugels, Regular Variation, Encyclopedia of Mathematics and its Applications, Cambridge University Press, 1987. doi:10.1017/CB09780511721434.

[26] A. Granata, The theory of higher-order types of asymptotic variation for differentiable functions. part i: Higher-order regular, smooth and rapid variation, Advances in Pure Mathematics 6 (12) (2016) 776.

[27] K. B. Athreya, S. N. Lahiri, Measure theory and probability theory, Springer Science \& Business Media, 2006.

[28] G. Samaey, T. Lelièvre, V. Legat, A numerical closure approach for kinetic models of polymeric fluids: exploring closure relations for FENE dumbbells, Computers \& Fluids 43 (1) (2011) $119-133$.

[29] B. Jørgensen, R. Labouriau, Exponential families and theoretical inference, Ph.D. thesis, University of Aarhus (1995).

[30] R. W. Keener, Theoretical statistics: Topics for a core course, Springer, 2011.

\section{AppendixA. Properties of cumulant generating functions}

First, we mention the convexity of $\mathcal{K}_{P}$.

Proposition 4 ([29, Thm. A.4]). Let $P \in \mathscr{P}^{d}$. Then

(i) The set $\Theta_{P}$ is convex.

(ii) $\mathcal{K}_{P}$ is a convex function on $\Theta_{P}$, and strictly convex if and only if $P$ is not concentrated in a single point.

When $X \sim P$ we also write $\mathcal{K}_{X}$ instead of $\mathcal{K}_{P}$, and $\Theta_{X}$ instead of $\Theta_{P}$.

Proposition 5 ([29, Thm. A.1 \& A.7], [30, 31]). Assume that $X \sim P$, where $P \in \mathscr{P}^{d}$. Then 
(i) $-\infty<\mathcal{K}_{P}(\theta) \leq+\infty$ for $\theta \in \mathbb{R}^{d}$.

(ii) $\mathcal{K}_{P}(0)=0$.

(iii) If $M \in \mathbb{R}^{l \times d}$ and $c \in \mathbb{R}^{l}$

$$
\begin{aligned}
\Theta_{M X+c} & =\left\{s \in \mathbb{R}^{l}: M^{\top} s \in \Theta_{X}\right\} \\
\mathcal{K}_{M X+c}(s) & =\mathcal{K}_{X}\left(M^{\top} s\right)+s \cdot c, \quad s \in \mathbb{R}^{l} .
\end{aligned}
$$

(iv) If $0 \in \operatorname{int} \Theta_{P}, \mathcal{K}_{P}$ is analytic on $\operatorname{int} \Theta_{P}$ with Taylor expansion around 0

$$
\mathcal{K}_{P}(\theta)=\sum_{j=1}^{+\infty} \frac{1}{j !} \mathrm{D}^{j} \mathcal{K}_{P}(0)[\theta]
$$

where $\mathrm{D}^{j} \mathcal{K}_{P}(0)$ is the $j$ th cumulant of $P$, considered as the $j$-linear mapping on $\mathbb{R}^{d}$, and $\mathrm{D}^{j} \mathcal{K}_{P}(0)[\theta] \doteq \mathrm{D}^{j} \mathcal{K}_{P}(0)[\theta, \ldots, \theta]$. In particular, $X$ has vector mean and covariance matrix

$$
\mathbb{E}[X]=\nabla \mathcal{K}_{P}(0), \quad \mathbb{V}(X)=\nabla^{2} \mathcal{K}_{P}(0) .
$$

(v) If $X, Y$ are independent, $\mathcal{K}_{X+Y}=\mathcal{K}_{X}+\mathcal{K}_{Y}$ and $\Theta_{X+Y}=\Theta_{X} \cap \Theta_{Y}$.

Proposition 6. If $Q=\mathcal{M}\left(\bar{\mu}^{s}, P\right)$ is the solution to 22 with a (slow) marginal mean $\bar{\mu}^{s} \in \mathbb{R}^{d_{s}}$ and a prior $P \in \mathscr{P}^{d}$, then

$$
\mathcal{K}_{Q}(\theta)=\mathcal{K}_{P}\left(\theta+\bar{\lambda}^{s} \oplus 0^{f}\right)-\mathcal{K}_{P}\left(\bar{\lambda}^{s} \oplus 0^{f}\right),
$$

where $\bar{\lambda}^{s} \in \mathbb{R}^{d_{s}}$ is a vector of Lagrange multipliers corresponding to $\bar{\mu}^{s}$ and $0^{f}=(0, \ldots, 0) \in \mathbb{R}^{d_{f}}$.

Proof. Using formula (2) we compute

$$
\begin{aligned}
\mathcal{K}_{Q}(\theta) & =\ln \mathbb{E}_{P}\left[e^{\theta \cdot \Pi} \frac{\mathrm{d} Q}{\mathrm{~d} P}\right] \\
& =\ln \mathbb{E}_{P}\left[e^{\theta \cdot \Pi+\bar{\lambda}^{s} \cdot \Pi^{s}} e^{-A\left(\bar{\lambda}^{s}, P^{s}\right)}\right] \\
& =\ln \mathbb{E}_{P}\left[e^{\left(\theta+\bar{\lambda}^{s} \oplus 0^{f}\right) \cdot \Pi}\right]-A\left(\bar{\lambda}^{s}, P^{s}\right) .
\end{aligned}
$$

It remains to note that by the definitions of log-partition function and the marginal distribution

$$
A\left(\bar{\lambda}^{s}, P^{s}\right)=\ln \mathbb{E}_{P^{s}}\left[e^{\bar{\lambda}^{s} \cdot \Pi^{s}}\right]=\ln \mathbb{E}_{P}\left[e^{\left(\bar{\lambda}^{s} \oplus 0^{f}\right) \cdot \Pi}\right] .
$$

Corollary 1. For any vectors $\bar{\mu}, \mu \in \mathbb{R}^{d}$ and a symmetric, non-negative definite matrix $\Sigma \in \mathbb{R}^{d \times d}$, $\mathcal{M}\left(\bar{\mu}, \mathcal{N}_{\mu, \Sigma}\right)=\mathcal{N}_{\bar{\mu}, \Sigma}$.

Proof. According to (3), the vector of Lagrange multipliers $\bar{\lambda}$ corresponding to matching with $\bar{\mu}$ satisfies $\nabla_{\lambda} A\left(\bar{\lambda}, \mathcal{N}_{\mu, \Sigma}\right)=\bar{\mu}$. When matching with mean only, the log-partition function coincides with the cumulant generating function, that is $A\left(\lambda, \mathcal{N}_{\mu, \Sigma}\right)=\mathcal{K}_{\mathcal{N}_{\mu, \Sigma}}(\lambda)$, and using formula from Example 1 we obtain

$$
\Sigma \bar{\lambda}=\bar{\mu}-\mu .
$$

Employing Proposition 6 with $Q=\mathcal{M}\left(\bar{\mu}, \mathcal{N}_{\mu, \Sigma}\right)$ we compute

$$
\begin{aligned}
\mathcal{K}_{Q}(\theta) & =\mu \cdot \theta+\frac{1}{2}\left(\theta^{\top} \Sigma \bar{\lambda}+\bar{\lambda}^{\top} \Sigma \theta\right)+\frac{1}{2} \theta^{\top} \Sigma \theta \\
& =\mu \cdot \theta+(\bar{\mu}-\mu) \cdot \theta+\frac{1}{2} \theta^{\top} \Sigma \theta \\
& =\bar{\mu} \cdot \theta+\frac{1}{2} \theta^{\top} \Sigma \theta .
\end{aligned}
$$

